Zhengping Wang · Huan-Song Zhou

\title{
Positive solutions for nonlinear Schrödinger equations with deepening potential well
}

Received March 30, 2007 and in revised form August 29, 2007

Abstract. Consider the following nonlinear Schrödinger equation:

$$
-\Delta u+(1+\lambda g(x)) u=f(u) \text { and } \quad u>0 \text { in } \mathbb{R}^{N}, \quad u \in H^{1}\left(\mathbb{R}^{N}\right), N \geq 3,
$$

where $\lambda \geq 0$ is a parameter, $g \in L^{\infty}\left(\mathbb{R}^{N}\right)$ vanishes on a bounded domain in $\mathbb{R}^{N}$, and the function $f$ is such that

$$
\lim _{s \rightarrow 0} f(s) / s=0 \quad \text { and } \quad 1 \leq \alpha+1=\lim _{s \rightarrow \infty} f(s) / s<\infty .
$$

We are interested in whether problem $(*)$ has a solution for any given $\alpha, \lambda \geq 0$. It is shown in [14] and [31] that problem $(*)$ has solutions for some $\alpha$ and $\lambda$. In this paper, we establish the existence of solution of $(*)$ for all $\alpha$ and $\lambda$ by using a variant of the Mountain Pass Theorem. Based on these results, we give a diagram in the $(\lambda, \alpha)$-plane showing how the solvability of problem $(*)$ depends on the parameters $\alpha$ and $\lambda$.

Keywords. Nonlinear Schödinger equations, mountain pass theorem, potential well, asymptotically linear

\section{Introduction}

In this paper, we are concerned with the existence of positive solutions for the following type of nonlinear Schrödinger equation:

$$
\left\{\begin{array}{l}
-\Delta u+V_{\lambda}(x) u=f(u) \quad \text { in } \mathbb{R}^{N}, \\
u \in H^{1}\left(\mathbb{R}^{N}\right), \quad N \geq 3,
\end{array}\right.
$$

where $V_{\lambda}(x)=1+\lambda g(x), \lambda>0$, and the functions $f$ and $g$ satisfy the following assumptions:

(F1) $f \in C\left(\mathbb{R}, \mathbb{R}^{+}\right), f(s) \equiv 0$ for all $s<0$ and $f(s) / s \rightarrow 0$ as $s \rightarrow 0^{+}$.

(F2) There exists $\alpha \geq 0$ such that $f(s) / s \rightarrow \alpha+1$ as $s \rightarrow \infty$.

(F3) $f(s) / s$ is nondecreasing in $s>0$.

Z. P. Wang, H. S. Zhou: Wuhan Institute of Physics and Mathematics, Academy of Sciences, P.O.Box 71010, Wuhan 430071, China; e-mail: wangzp@wipm.ac.cn; hszhou@wipm.ac.cn

Mathematics Subject Classification (2000): 35J20, 35J60, 35J10 
(G1) $g \in L^{\infty}\left(\mathbb{R}^{N}, \mathbb{R}\right)$ and there exists a nonempty bounded smooth domain $\Omega \subset \mathbb{R}^{N}$ such that

$$
g(x) \equiv 0 \text { on } \bar{\Omega}, \quad g(x) \in(0,1] \text { on } \mathbb{R}^{N} \backslash \bar{\Omega} \quad \text { and } \quad \lim _{|x| \rightarrow \infty} g(x)=1 .
$$

Remark 1.1. $f(s)$ with property (F2) is usually called asymptotically linear in $s$ at infinity. Let $F(t)=\int_{0}^{t} f(s) d s$. Condition (F3) implies that for any constant $D \geq 1, t \in[0,1]$ and $s \geq 0$,

$$
\begin{aligned}
& 0 \leq \frac{1}{2} t^{2} f(s) s-F(t s) \leq D\left[\frac{1}{2} f(s) s-F(s)\right], \quad \text { or } \\
& 0 \leq \frac{1}{2} f(t s) t s-F(t s) \leq D\left[\frac{1}{2} f(s) s-F(s)\right] .
\end{aligned}
$$

Condition (F3) is only used to get the condition 1.2 or 1.3 , which is required in proving the boundedness of a (PS) sequence in Section 3. Therefore, we may slightly weaken (F3) by simply assuming (1.2) or 1.3) for some $D \geq 1$ as in [6, 17].

By condition (G1), the potential $V_{\lambda}$ has a potential well with bottom $\Omega$ and depth controlled by the parameter $\lambda$.

Notation. Throughout this paper, we denote by $\xi_{1}$ the first eigenvalue of the Dirichlet problem

$$
\left\{\begin{array}{l}
-\Delta \varphi=\xi \varphi \quad \text { in } \Omega, \quad \Omega \text { is given by }(\mathrm{G} 1), \\
\varphi \in H_{0}^{1}(\Omega),
\end{array}\right.
$$

and let $\varphi_{1}>0$ be a normalized $\xi_{1}$-eigenfunction. Denote by $\|\cdot\|$ and $|\cdot|_{p}$, respectively, the standard norms of $H^{1}\left(\mathbb{R}^{N}\right)$ and $L^{p}\left(\mathbb{R}^{N}\right)$ with $1 \leq p \leq \infty$. Let $\lambda=\Lambda(\alpha)$ be the principal eigenvalue of the linear eigenvalue problem

$$
-\Delta u-\alpha u+\lambda g(x) u=0, \quad \alpha>0 .
$$

It is proved in [32] that $\Lambda(\alpha)$ always exists if $\alpha \in\left(\Gamma, \xi_{1}\right)$ with

$$
\Gamma=\inf \left\{\int_{\mathbb{R}^{N}}|\nabla u|^{2} d x: u \in H^{1}\left(\mathbb{R}^{N}\right) \text { and } \int_{\mathbb{R}^{N}}(1-g) u^{2} d x=1\right\},
$$

and the $\Lambda(\alpha)$-eigenfunction $\varphi_{\Lambda}$ is the only eigenfunction which does not change sign. Moreover, $\Lambda(\alpha)>\alpha$, and $\Lambda(\alpha)$ is the greatest eigenvalue of (1.5).

If $f(s)$ is superlinear in $s$ at infinity, i.e. $\alpha=\infty$ in (F2), problem (1.1) for $\lambda$ large, that is, when $V_{\lambda}$ has a steep potential well, has been widely studied (see, for example, [25, 2, 3, 7, 12, 10, 14, 1, 22, 19, 5] etc.). If $f(s)$ is asymptotically linear, i.e. $\alpha<\infty$ in (F2), problem (1.1) was studied by van Heerden and Wang [14], who proved that problem (1.1) has a positive solution (or multiple solutions for odd $f$ ) if $\alpha>\xi_{1}$ and $\lambda$ is large. The multiple existence results of [14] were extended to more general cases [13, 22], in which $\alpha>\xi_{1}$ may not be satisfied. If $f(s)$ is sublinear, some existence results for 1.1 were obtained recently in [33]. Moreover, we mention that the asymptotically linear problem 
1.1) with $V_{\lambda}(x) \equiv V(x)$ was also discussed by many authors, for example, Jeanjean and Tanaka [16, 17], Liu and Wang [24, 23] et al., even if $V(x)$ vanishes at infinity [21]. As pointed out in [31], the results of [16, 24] imply that (1.1) may have a positive solution for $\lambda$ not necessarily large. In our notation, the existence results for (1.1) proved in [16] and [24] are based on the following conditions:

(i) $\alpha+1>\inf \sigma\left(-\Delta+V_{\lambda}\right)$

(ii) there exists $\delta>0$ such that

$$
\lambda+1-\delta>\frac{2}{s^{2}} \int_{0}^{s} f(t) d t \quad \text { for all } s>0,
$$

where $\sigma\left(-\Delta+V_{\lambda}\right)$ denotes the spectrum of the Schrödinger operator $-\Delta+V_{\lambda}$ in $L^{2}\left(\mathbb{R}^{N}\right)$.

In fact, condition (i) implies that $\lambda<\Lambda(\alpha)$, and (ii) gives that $\lambda>\alpha$.

Very recently, under these conditions the authors of [31] showed that problem (1.1) has a positive solution for any $\lambda$ in the interval $(\alpha, \Lambda(\alpha))$ with $\Gamma<\alpha<\xi_{1}$, while 1.1 . has no positive solution if $(\lambda, \alpha) \in A \cup B$ with

$$
A=\{(\lambda, \alpha): \alpha \leq \Gamma \text { and } \lambda \geq \alpha\}, \quad B=\left\{(\lambda, \alpha): \Gamma<\alpha<\xi_{1} \text { and } \lambda \geq \Lambda(\alpha)\right\} .
$$

With the above mentioned results, we can draw a diagram in the $(\lambda, \alpha)$-plane (see Figure 1) below, where $\lambda=\Lambda(\alpha)$ is a curve which is shown in [31] such that $\Lambda(\alpha) \rightarrow \Gamma$ as $\alpha \rightarrow \Gamma^{+}$and $\Lambda(\alpha) \rightarrow \infty$ as $\alpha \rightarrow \xi_{1}^{-}$. From Figure 1, it is clear that problem 1.1 . has always a positive solution in region $\mathrm{I}=\left\{(\lambda, \alpha): \lambda>\alpha\right.$ is large and $\left.\alpha>\xi_{1}\right\}$ by [14] and also in region II $=\left\{(\lambda, \alpha): \lambda \in(\alpha, \Lambda(\alpha))\right.$ with $\left.\alpha \in\left(\Gamma, \xi_{1}\right)\right\}$ by [31]. Problem [1.1] has no positive solution in regions III $\cup$ IV $=B \cup A$ by [31]. It is natural to ask whether there is any positive solution for problem $(1.1)$ in regions V, VI and VII as well as the corresponding boundaries. In this paper, we answer this question completely and we find that there is a boundary, denoted by

$$
\ell=\{(\lambda, \alpha): \lambda=\alpha \text { and } \alpha \in[0, \Gamma]\} \cup\{(\lambda, \alpha): \lambda=\Lambda(\alpha)\},
$$

which splits the first quadrant of the $(\lambda, \alpha)$-plane into two parts such that problem (1.1) has no positive solution for $(\lambda, \alpha)$ below $\ell$ (including $\ell$ itself and the positive $\lambda$-axis, see the region marked with $\times$ in Figure 1), and problem (1.1) has always a positive solution for $(\lambda, \alpha)$ above $\ell$ (including the positive $\alpha$-axis). Moreover, just using the Mountain Pass Theorem we also give a simple proof for the existence of a positive solution to problem 1.1) in regions I and II, although these results were essentially contained in [14, 31]. Therefore, it is now clear how the existence of positive solutions of problem (1.1) depends on the parameters $\lambda$ and $\alpha$. In other words, for any given $\alpha \geq 0$ and $\lambda \geq 0$, to see whether problem (1.1) has a positive solution we need only check on which side of the curve $\ell$ the point $(\lambda, \alpha)$ is located.

In [31], the authors proved that a positive solution obtained in region II $=\{(\lambda, \alpha)$ : $\left.\lambda \in(\alpha, \Lambda(\alpha)), \alpha \in\left(\Gamma, \xi_{1}\right)\right\}$ is bounded away from zero when $\lambda \rightarrow \alpha^{+}$and blows up as $\lambda \rightarrow \Lambda(\alpha)^{-}$. In this paper, we also prove that in regions VI and VII, the solution is 


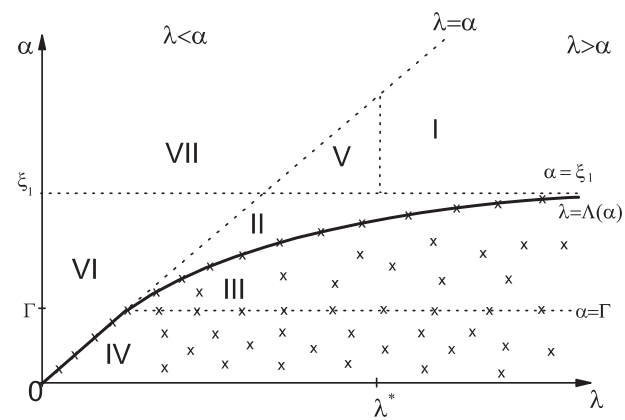

Fig. 1. $\ell$ is the bold curve, which is the boundary between the existence and nonexistence.

bounded away from zero and bounded above for all $\lambda \in\left(0, \lambda_{0}\right]$ and each fixed $\lambda_{0}<\alpha$. However, the behavior of the solutions when $\lambda$ is close to $\alpha$ on both sides of the line $\lambda=\alpha$ is still open. Do the limits of the solutions from both sides of $\lambda=\alpha$ coincide?

Here are the main results of this paper.

Theorem 1.1 (Existence). Suppose that (F1), (F2), (F3), (G1) hold, and $\lambda$ and $\alpha$ satisfy one of the following five possibilities:

(i) $\alpha>0$ and $0<\lambda<\alpha$, i.e., regions VI and VII in Figure 1.

(ii) $\lambda=\alpha$ and $\alpha>\Gamma$, where $\Gamma$ is given by (1.6).

(iii) $\alpha>\xi_{1}$ and $\lambda \in(0, \infty)$, i.e., regions I, V and VII in Figure 1.

(iv) $\alpha=\xi_{1}$ and $\lambda \in(\alpha, \infty)$.

(v) $\lambda \in(\alpha, \Lambda(\alpha))$ and $\alpha \in\left(\Gamma, \xi_{1}\right)$, i.e., region II in Figure 1.

Then problem (1.1) has a positive solution.

Remark 1.2. (1) For $\lambda>0$ large, case (iii) was proved in [14]. Case (v) was essentially shown in [31] by bifurcation theory. The aim of this paper is to prove Theorem 1.1] in all cases (i)-(v) simply by a variant of the Mountain Pass Theorem.

(2) Condition (F3) of this theorem can be replaced by assuming (1.2) or (1.3) for some $D \geq 1$.

Theorem 1.2. Suppose that (F1), (F2), (G1) hold and $\lambda=0$. Then problem (1.1) has a positive solution for any $\alpha>0$.

Theorem 1.3 (Nonexistence). Suppose that (F1), (F2), (F3), (G1) hold and $\alpha=0$. Then problem (1.1) has no positive solution for any $\lambda \geq 0$.

Theorem 1.4. For any $\alpha>0$ and $\lambda_{0} \in(0, \alpha)$, there exist $C_{1}, C_{2}>0$, depending on $\alpha$ and $\lambda_{0}$, such that

$$
C_{2} \leq\left\|u_{\lambda}\right\| \leq C_{1} \quad \text { for all } \lambda \in\left(0, \lambda_{0}\right]
$$


where $u_{\lambda}$ is the positive solution of (1.1) given by Theorem 1.1. If $g(x)$ is radial, then

$$
u_{\lambda} \rightarrow u_{0} \quad \text { strongly in } H^{1}\left(\mathbb{R}^{N}\right) \text { as } \lambda \rightarrow 0^{+},
$$

and $u_{0} \not \equiv 0$ solves the equation

$$
-\Delta u+u=f(u), \quad u \in H^{1}\left(\mathbb{R}^{N}\right) .
$$

It is well known that a positive solution of problem 1.1 corresponds to a nonzero critical point of the energy functional $I$ defined on $H^{1}\left(\mathbb{R}^{N}\right)$ by

$$
I(u)=\frac{1}{2} \int_{\mathbb{R}^{N}}|\nabla u|^{2}+(1+\lambda g(x)) u^{2} d x-\int_{\mathbb{R}^{N}} F(u) d x,
$$

where $F(t)=\int_{0}^{t} f(s) d s$. It is easy to see that $I$ is $C^{1}$ under our conditions (F1), (F2), (G1). Instead of using abstract critical point theorems, we seek critical points of $I$ by simply using the following version of the Mountain Pass Theorem, applied in [8, 16].

Proposition 1.1 (Mountain Pass Theorem). Let E be a real Banach space with dual $E^{*}$, and $I \in C^{1}(E, \mathbb{R})$ with $I(0)=0$ be such that

$$
I(u) \geq 0 \quad \text { for all } u \in E \text { with }\|u\| \leq \rho, \quad \inf _{\|u\|=\rho} I(u) \geq \alpha \quad \text { and } \quad I(e)<0,
$$

for some $\rho, \alpha>0$, and $e \in E$ with $\|e\|>\rho$. Define

$$
c=\inf _{\gamma \in \Gamma} \max _{0 \leq t \leq 1} I(\gamma(t)),
$$

where $\Gamma=\{\gamma \in C([0,1], E): \gamma(0)=0, I(\gamma(1))<0\}$. Then there exists a sequence $\left\{u_{n}\right\} \subset E$ such that

$$
I\left(u_{n}\right) \stackrel{n}{\rightarrow} c \geq \alpha \quad \text { and } \quad\left(1+\left\|u_{n}\right\|\right)\left\|I^{\prime}\left(u_{n}\right)\right\|_{E^{*}} \stackrel{n}{\rightarrow} 0 .
$$

A more general version of Proposition 1.1 can be found in Chapter IV of [11] or in [26], for example. Based on [11], a simple proof to Proposition 1.1 was given by Stuart [28]. A sequence with properties (1.8) is usually called a Cerami sequence at level $c,(\mathrm{Ce})_{c}$ sequence for short. Clearly, a $(\mathrm{Ce})_{c}$ sequence is also a usual $(\mathrm{PS})_{c}$ sequence.

Under the assumptions of Theorem 1.1 and using the properties of the eigenfunctions $\varphi_{1}$ in 1.4 ) and $\varphi_{\Lambda}$ in (1.5), it is not difficult to verify that the function $I$ defined by 1.7) satisfies all the conditions of the above version of Mountain Pass Theorem with $E=H^{1}\left(\mathbb{R}^{N}\right)$; in fact, (F3) is not necessary for this purpose (see Lemmas 2.1 and 2.2). Then Proposition 1.1 guarantees that there is a Cerami sequence $\left\{u_{n}\right\} \subset H^{1}\left(\mathbb{R}^{N}\right)$ which has the properties (1.8). To get a positive solution of problem (1.1), it is sufficient to show first that $\left\{u_{n}\right\}$ is bounded in $H^{1}\left(\mathbb{R}^{N}\right)$ and then that it converges to a nonzero critical point of $I$. Since the nonlinear term $f(s)$ is asymptotically linear, not superlinear, at infinity, it is known that to prove $\left\{u_{n}\right\}$ is bounded in $H^{1}\left(\mathbb{R}^{N}\right)$ is usually not easy, although many efforts having been made in recent years [29, 15, 30]. Roughly speaking, here are the main ideas. Suppose that $\left\{u_{n}\right\}$ is not bounded, that is, $\left\|u_{n}\right\| \stackrel{n}{\rightarrow} \infty$. Then $w_{n}=u_{n} /\left\|u_{n}\right\|$ 
is bounded in $H^{1}\left(\mathbb{R}^{N}\right)$ and there is $w \in H^{1}\left(\mathbb{R}^{N}\right)$ such that $w_{n} \stackrel{n}{\rightarrow} w$ weakly in $H^{1}\left(\mathbb{R}^{N}\right)$. Next, we try to use the properties (1.8) to get a contradiction via $w$. Motivated by [15], we seek a contradiction by applying the following variant of the concentration-compactness lemma [35. Lemma 2.1], essentially based on [20]. This kind of idea was used in many papers, for example, [16, 18, 14].

Lemma 1.1 ([35, Lemma 2.1]). For $R>0$, set $B_{R}=\left\{x \in \mathbb{R}^{N}:|x| \leq R\right\}$. Let $\left\{\rho_{n}\right\}$ be a sequence in $L^{1}\left(\mathbb{R}^{N}\right)$ satisfying

$$
\rho_{n} \geq 0 \quad \text { on } \mathbb{R}^{N}, \quad \lim _{n \rightarrow \infty} \int_{\mathbb{R}^{N}} \rho_{n} d x=\eta \quad \text { for some } \eta>0 .
$$

Then there exists a subsequence of $\left\{\rho_{n}\right\}$, still denoted by $\left\{\rho_{n}\right\}$, satisfying one of the following two conditions:

(i) (Vanishing) $\lim _{n \rightarrow \infty} \sup _{y \in \mathbb{R}^{N}} \int_{B_{R}(y)} \rho_{n} d x=0$ for all $R>0$.

(ii) (Nonvanishing) There exist $v \in(0, \eta), R<\infty$ and $\left\{y_{n}\right\} \subset \mathbb{R}^{N}$ such that

$$
\lim _{n \rightarrow \infty} \int_{B_{R}\left(y_{n}\right)} \rho_{n} d x \geq v>0 .
$$

To get a contradiction, the main idea is to apply Lemma 1.1 with $\rho_{n}=w_{n}^{2}$ and prove that neither of the above cases can occur. To rule out Vanishing is somehow standard and not difficult; it was done in [14] by an estimate for $\lambda$ large (see Lemma 2.3 of [14]), but in our cases $\lambda$ may be small, so the condition (1.2) or $[1.3]$ is assumed. However, to rule out Nonvanishing does not seem easy and needs some more elaborate results on the linear eigenvalue problem (1.5). In fact, if Nonvanishing occurs, then we can show that the weak limit $w$ of the sequence $\left\{w_{n}\right\}$ (or its translation) in $H^{1}\left(\mathbb{R}^{N}\right)$ is nonzero and satisfies either

$$
\text { (a) : }-\Delta w+(1+\lambda) w=(1+\alpha) w \text {, or } \quad \text { (b): the equation 1.5. }
$$

It is clear that (a) is impossible since $-\Delta$ has no nontrivial eigenvalue in $L^{2}\left(\mathbb{R}^{N}\right)$. Thanks to the results of [16] and [32], we are able to show that (b) is also impossible under the assumptions of Theorem 1.1 .

To get a solution for problem 1.1, the last step is to prove that the bounded sequence $\left\{u_{n}\right\}$ converges to a nontrivial critical point of $I$. It is known that $\left\{u_{n}\right\}$ always converges weakly to a critical point $u$ of $I$, but usually it is not clear whether $u \neq \equiv 0$. In most cases one can use the concentration-compactness principle [20] to prove that $\left\{u_{n}\right\}$ is strongly convergent, that is, I satisfies the (PS) condition (see e.g. [23], [8], [18]); then $u \neq \equiv 0$ by the mountain pass properties of $I$. However, it is not always easy to check the (PS) condition, especially in some cases of our problem, for example, when $\lambda=\alpha$. Motivated by [16], instead of proving the (PS) condition by the concentration-compactness procedure (although we may prove it in most cases of Theorem 1.1, see Proposition 4.1), in Section 4 we prove Theorem 1.1 by showing that $u \neq \equiv 0$ in a simple way. Very recently, $\mathrm{Liu}, \mathrm{Su}$ and Weth in [23] established a compactness result for a similar problem to (1.1), which is helpful for many kinds of problems, but their condition (A4) may not be satisfied in all our cases. 


\section{Preliminary results and properties of $I$}

In this section, we first recall some properties of the Schrödinger operator $-\Delta+V(x)$, based on [24, 16, 32]; they will be used in the following sections. Then we prove some lemmas on the energy functional $I$ associated with problem (1.1), which are required when applying the Mountain Pass Theorem.

Proposition 2.1. Suppose that $V \in L^{\infty}\left(\mathbb{R}^{N}, \mathbb{R}^{+}\right)$and there is a constant $V_{\infty} \in(0, \infty)$ such that $\lim _{|x| \rightarrow \infty} V(x)=V_{\infty}$. Define

$$
\mu^{*}:=\inf \sigma(-\Delta+V)=\inf _{0 \neq u \in H^{1}\left(\mathbb{R}^{N}\right)} \frac{\int_{\mathbb{R}^{N}}\left[|\nabla u|^{2}+V(x) u^{2}\right] d x}{\int_{\mathbb{R}^{N}} u^{2} d x} .
$$

We have:

(i) If $\mu^{*}<V_{\infty}$, then there is $\phi^{*} \in H^{1}\left(\mathbb{R}^{N}\right)$ with $\phi^{*}>0$ in $\mathbb{R}^{N}$ such that

$$
-\Delta \phi^{*}+V(x) \phi^{*}=\mu^{*} \phi^{*}
$$

(ii) For any $\mu>\mu^{*}$, there is no nonnegative $\phi \in H^{1}\left(\mathbb{R}^{N}\right), \phi \not \equiv 0$, such that

$$
-\Delta \phi+V(x) \phi=\mu \phi \text {. }
$$

Proof. Part (i) was essentially proved in [24], but here we do not require that $V(x)$ is bounded below by a positive constant and we give a simple proof motivated by [34].

It is known that to prove (i) we need only show that the infimum $\mu^{*}$ can be attained by a positive function $\phi^{*} \in H^{1}\left(\mathbb{R}^{N}\right)$. For this purpose, let $\left\{u_{n}\right\} \subset H^{1}\left(\mathbb{R}^{N}\right)$ be such that

$$
\int_{\mathbb{R}^{N}}\left[\left|\nabla u_{n}(x)\right|^{2}+V(x) u_{n}^{2}\right] d x \stackrel{n}{\rightarrow} \mu^{*} \quad \text { and } \quad \int_{\mathbb{R}^{N}}\left|u_{n}(x)\right|^{2} d x=1 .
$$

Clearly, $\left\{u_{n}\right\}$ is bounded in $H^{1}\left(\mathbb{R}^{N}\right)$ since $0 \leq V \in L^{\infty}\left(\mathbb{R}^{N}\right)$. So, we may assume that, for some $u \in H^{1}\left(\mathbb{R}^{N}\right)$,

$$
u_{n} \stackrel{n}{\rightarrow} u \quad \text { weakly in } H^{1}\left(\mathbb{R}^{N}\right), \quad u_{n} \stackrel{n}{\rightarrow} u \quad \text { strongly in } L_{\text {loc }}^{2}\left(\mathbb{R}^{N}\right) .
$$

Let $\tilde{V}(x)=V(x)-V_{\infty}$, so that $\tilde{V}(x) \rightarrow 0$ as $|x| \rightarrow \infty$. Then by 2.1 it is not difficult to see that

$$
\lim _{n \rightarrow \infty} \int_{\mathbb{R}^{N}} \tilde{V}(x)\left|u_{n}(x)\right|^{2} d x=\int_{\mathbb{R}^{N}} \tilde{V}(x)|u(x)|^{2} d x .
$$

Define

$$
J(v) \triangleq \int_{\mathbb{R}^{N}}\left[|\nabla v(x)|^{2}+\tilde{V}(x) v^{2}\right] d x
$$

Then

$$
J(u) \leq \lim _{n \rightarrow \infty} J\left(u_{n}\right)=\mu^{*}-V_{\infty}<0 .
$$


On the other hand, by the definition of $\mu^{*}$ we see that

$$
J(u) \geq\left[\mu^{*}-V_{\infty}\right] \int_{\mathbb{R}^{N}}|u(x)|^{2} d x .
$$

These inequalities imply that $\int_{\mathbb{R}^{N}} u^{2}(x) d x \geq 1$. Therefore, $\int_{\mathbb{R}^{N}} u^{2}(x) d x=1$ by 2.1 . So, $J(u)=\mu^{*}-V_{\infty}$ and $u \in H^{1}\left(\mathbb{R}^{N}\right)$ attains $\mu^{*}$. The strong maximum principle implies that $u>0$ in $\mathbb{R}^{N}$.

Part (ii) was proved in [16]. For the sake of completeness, we give a sketch of its proof. Argue by contradiction. Suppose that $u \geq 0,0 \not \equiv \in \in H^{1}\left(\mathbb{R}^{N}\right)$ solves

$$
-\Delta u+V(x) u=\mu u \text {. }
$$

For $R>0$, define

$$
\mu_{R}=\inf \left\{\int_{B_{R}}\left[|\nabla u|^{2}(x)+V(x) u^{2}(x)\right] d x: \int_{B_{R}} u^{2}(x) d x=1, u \in H_{0}^{1}\left(B_{R}\right)\right\} .
$$

It is not difficult to see that there exists a $w_{R} \in H_{0}^{1}\left(B_{R}\right) \backslash\{0\}$, with $w_{R} \geq 0$ and $\int_{B_{R}} w_{R}^{2}(x) d x=1$, such that

$$
-\Delta w_{R}+V(x) w_{R}=\mu_{R} w_{R}, \quad x \in B_{R},
$$

and the strong maximum principle implies that

$$
w_{R}(x)>0, \quad \forall x \in B_{R}, \quad \frac{\partial w_{R}(x)}{\partial v}<0, \quad \forall|x|=R .
$$

It follows from 2.3 that

$$
\begin{aligned}
\mu_{R} \int_{B_{R}} w_{R} u d x & =\int_{B_{R}} \nabla u \nabla w_{R}+\int_{B_{R}} V(x) u w_{R} d x-\int_{\partial B_{R}} \frac{\partial w_{R}}{\partial v} u d \sigma \\
& =\int_{B_{R}} \mu u w_{R} d x-\int_{\partial B_{R}} \frac{\partial w_{R}}{\partial v} u d \sigma \quad \text { by using (2.2) } \\
& \geq \mu \int_{B_{R}} u w_{R} d x \quad \text { by 2.4). }
\end{aligned}
$$

This implies that $\mu_{R} \geq \mu$, since we can choose $R>0$ large enough such that $\int_{B_{R}} u w_{R} d x$ $>0$ by noting that $u \geq(\not \equiv) 0$ and $w_{R}>0$.

On the other hand, as $\mu>\mu^{*}$, there is a constant $v$ such that $\mu^{*}<v<\mu$. The definition of $\mu^{*}$ implies there exists $v \in H^{1}\left(\mathbb{R}^{N}\right)$ such that $\int_{R^{N}} v^{2}(x) d x=1$ and

$$
\mu^{*} \leq \int_{\mathbb{R}^{N}}\left[|\nabla v|^{2}+V(x) v^{2}\right] d x<v .
$$

Since $C_{0}^{\infty}\left(\mathbb{R}^{N}\right)$ is dense in $H^{1}\left(\mathbb{R}^{N}\right)$, we may assume $v \in C_{0}^{\infty}\left(\mathbb{R}^{N}\right)$ and choose $R>0$ large enough such that supp $v \subset B_{R}$. By the definition of $\mu_{R}$, we have

$$
\mu_{R} \leq \int_{\mathbb{R}^{N}}\left[|\nabla v|^{2}+V(x) v^{2}\right] d x<v<\mu .
$$

This is a contradiction. 
Proposition 2.2 ([32, Theorem 1.5]). Suppose that condition (G1) holds.

(i) If $\alpha \geq \xi_{1}$, then the eigenvalue problem

$$
-\Delta u+\lambda g(x) u=\alpha u, \quad u \in H^{1}\left(\mathbb{R}^{N}\right),
$$

has no eigenvalue $\lambda \in[\alpha, \infty)$ with a nonnegative eigenfunction.

(ii) If $\Gamma<\alpha<\xi_{1}$, then there exists a unique eigenvalue $\lambda=\Lambda(\alpha)$ of $(1.5)$ having $a$ positive eigenfunction. All other eigenvalues of $(1.5)$ are less than $\Lambda(\alpha)$ and their eigenfunctions change sign.

Remark 2.1. If $\alpha \geq \xi_{1}$ and $\lambda>\alpha$, we must have

$$
\alpha \neq \inf _{0 \neq u \in H^{1}\left(\mathbb{R}^{N}\right)} \frac{\int_{\mathbb{R}^{N}}\left[|\nabla u|^{2}+\lambda g(x) u^{2}\right] d x}{\int_{\mathbb{R}^{N}} u^{2} d x} .
$$

See the proof of Lemma 3.3(ii) in [32]. Otherwise, the conclusions of Proposition 2.1.i) and Proposition 2.2 (i) are contradictory.

Lemma 2.1. Assume that (F1), (F2), (G1) hold. Then for any $\alpha, \lambda \in(0, \infty)$ there exist $\rho, \beta>0$ such that $I(u) \geq 0$ for all $u \in H^{1}\left(\mathbb{R}^{N}\right)$ with $0<\|u\| \leq \rho$, and

$$
I(u) \geq \beta>0 \quad \text { for all } u \in H^{1}\left(\mathbb{R}^{N}\right) \text { with }\|u\|=\rho .
$$

Proof. It follows from (F1) and (F2) that for any $\epsilon>0$, there exist $p \in\left(1, \frac{N+2}{N-2}\right)$ and $A=A(\epsilon, p)>0$ such that for all $s>0$,

$$
F(s) \leq \frac{1}{2} \epsilon s^{2}+A s^{p+1} .
$$

For any $\alpha>0$ and $0<\lambda<\infty$, by (G1) and Sobolev inequalities we have

$$
\begin{aligned}
I(u) & \geq \frac{1}{2} \int_{\mathbb{R}^{N}}\left[|\nabla u|^{2}+u^{2}\right] d x-\epsilon \int_{\mathbb{R}^{N}} u^{2} d x-A(\epsilon) \int_{\mathbb{R}^{N}}|u|^{p+1} d x \\
& \geq \frac{1}{2}\|u\|^{2}-C_{1} \epsilon\|u\|^{2}-C_{2}(\epsilon)\|u\|^{p+1} .
\end{aligned}
$$

So, the lemma is proved by taking $\epsilon=1 / 4 C_{1}$ and $\rho>0$ small enough.

Lemma 2.2. Assume that (F1), (F2), (G1) hold and $\alpha, \lambda>0$ satisfy one of the following conditions:

(i) $\lambda \in(0, \alpha)$.

(ii) $\lambda=\alpha$ and $\alpha>\Gamma$.

(iii) $\alpha>\xi_{1}$ and $\lambda \in(0, \infty)$.

(iv) $\alpha=\xi_{1}$ and $\lambda \in(\alpha, \infty)$.

(v) $\alpha<\xi_{1}$ and $\lambda \in(\alpha, \Lambda(\alpha))$.

Then, for $\rho$ given by Lemma 2.1 there exists $e \in H^{1}\left(\mathbb{R}^{N}\right)$ with $\|e\|>\rho$ such that $I(e)<0$. 
Proof. (i) Since $0<\lambda<\alpha$ and

$$
\inf \left\{\int_{\mathbb{R}^{N}}|\nabla u|^{2} d x: u \in H^{1}\left(\mathbb{R}^{N}\right) \text { with } \int_{\mathbb{R}^{N}} u^{2} d x=1\right\}=0,
$$

there exists $\phi_{1} \in H^{1}\left(\mathbb{R}^{N}\right) \backslash\{0\}$ such that

$$
\int_{\mathbb{R}^{N}}\left|\nabla \phi_{1}\right|^{2} d x<(\alpha-\lambda) \int_{\mathbb{R}^{N}}\left|\phi_{1}\right|^{2} d x .
$$

Clearly, we may assume that $\phi_{1} \geq 0$ on $\mathbb{R}^{N}$. Hence, by Fatou's lemma we have

$$
\begin{aligned}
\lim _{t \rightarrow \infty} \frac{I\left(t \phi_{1}\right)}{t^{2}} & =\frac{1}{2} \int_{\mathbb{R}^{N}}\left[\left|\nabla \phi_{1}\right|^{2}+(1+\lambda g) \phi_{1}^{2}\right] d x-\lim _{t \rightarrow \infty} \int_{\mathbb{R}^{N}} \frac{F\left(t \phi_{1}\right)}{t^{2} \phi_{1}^{2}} \phi_{1}^{2} d x \\
& \leq \frac{1}{2} \int_{\mathbb{R}^{N}}\left[\left|\nabla \phi_{1}\right|^{2}+(1+\lambda g) \phi_{1}^{2}-(1+\alpha) \phi_{1}^{2}\right] d x \\
& \leq \frac{1}{2} \int_{\mathbb{R}^{N}}\left[\left|\nabla \phi_{1}\right|^{2}+(\lambda-\alpha) \phi_{1}^{2}\right] d x<0 .
\end{aligned}
$$

So, in this case the conclusion is proved by choosing $e=t \phi_{1}$ for $t$ large.

(ii) In this case, $\alpha>\Gamma$ and $\lambda=\alpha$. By the definition of $\Gamma$ in $(1.6)$, there is $\phi_{2} \in$ $H^{1}\left(\mathbb{R}^{N}\right) \backslash\{0\}$ with $\phi_{2} \geq 0$ on $\mathbb{R}^{N}$ such that

$$
\int_{\mathbb{R}^{N}}\left|\nabla \phi_{2}\right|^{2} d x<\alpha \int_{\mathbb{R}^{N}}(1-g(x))\left|\phi_{2}\right|^{2} d x .
$$

Then, with this property we see that 2.6 still holds for $\phi_{2}$ and the conclusion can be proved by taking $e=t \phi_{2}$ for $t>0$ large.

(iii) Let $\varphi_{1} \in H_{0}^{1}(\Omega)$ be an eigenfunction corresponding to $\xi_{1}$ with $\int_{\Omega} \varphi_{1}^{2} d x=1$ (see (1.4)). Let $\tilde{\varphi} \in H^{1}\left(\mathbb{R}^{N}\right)$ be such that $\tilde{\varphi}(x) \equiv \varphi_{1}(x)$ for $x \in \Omega$ and $\tilde{\varphi}(x) \equiv 0$ for $x \in \Omega^{c}$. Then it is easy to see that

$$
g(x) \tilde{\varphi}(x) \equiv 0 \quad \text { for all } x \in \mathbb{R}^{N} .
$$

This and $\alpha>\xi_{1}$ imply that 2.6 still holds for $\tilde{\varphi}$, and the lemma is proved by taking $e=t \tilde{\varphi}$ for $t>0$ large.

(iv) Setting

$$
\mu_{\lambda}:=\inf \sigma\left(-\Delta+V_{\lambda}\right)=\inf _{0 \neq u \in H^{1}\left(\mathbb{R}^{N}\right)} \frac{\int_{\mathbb{R}^{N}}\left[|\nabla u|^{2}+V_{\lambda}(x) u^{2}\right] d x}{\int_{\mathbb{R}^{N}} u^{2} d x} .
$$

Let $\tilde{\varphi}$ be given by the proof of (iii). Then for any $\lambda>0$,

$$
\mu_{\lambda} \leq \frac{\int_{\mathbb{R}^{N}}\left[|\nabla \tilde{\varphi}|^{2}+V_{\lambda}(x) \tilde{\varphi}^{2}\right] d x}{\int_{\mathbb{R}^{N}} \tilde{\varphi}^{2} d x}=\frac{\int_{\mathbb{R}^{N}}\left[|\nabla \tilde{\varphi}|^{2}+\tilde{\varphi}^{2}\right] d x}{\int_{\mathbb{R}^{N}} \tilde{\varphi}^{2} d x}=1+\xi_{1} .
$$


We claim that $\mu_{\lambda}<1+\xi_{1}$ for any $\lambda>\alpha$. Indeed, if there exists $\lambda_{0}>\alpha$ such that $\mu_{\lambda_{0}}=1+\xi_{1}$, then $\mu_{\lambda_{0}}<1+\lambda_{0}$ since $\alpha=\xi_{1}$ in this case. By Proposition 2.11(i) there is a positive eigenfunction $\phi \in H^{1}\left(\mathbb{R}^{N}\right)$ satisfying

$$
-\Delta \phi+\left(1+\lambda_{0} g(x)\right) \phi=\left(1+\xi_{1}\right) \phi .
$$

But by Proposition 2.2(i) this is impossible. Thus for $\alpha=\xi_{1}$ and any $\lambda \in(\alpha, \infty)$, we have $\mu_{\lambda}<1+\alpha$. Then it follows from the definition of $\mu_{\lambda}$ that there is a nonnegative function $\phi_{0} \in H^{1}\left(\mathbb{R}^{N}\right) \backslash\{0\}$ satisfying

$$
\int_{\mathbb{R}^{N}}\left[\left|\nabla \phi_{0}\right|^{2}+V_{\lambda}(x) \phi_{0}^{2}\right] d x<(1+\alpha) \int_{\mathbb{R}^{N}} \phi_{0}^{2} d x .
$$

From Fatou's lemma we get

$$
\begin{aligned}
\lim _{t \rightarrow \infty} \frac{I\left(t \phi_{0}\right)}{t^{2}} & =\frac{1}{2} \int_{\mathbb{R}^{N}}\left[\left|\nabla \phi_{0}\right|^{2}+(1+\lambda g) \phi_{0}^{2}\right] d x-\lim _{t \rightarrow \infty} \int_{\mathbb{R}^{N}} \frac{F\left(t \phi_{0}\right)}{t^{2} \phi_{0}^{2}} \phi_{0}^{2} d x \\
& \leq \frac{1}{2} \int_{\mathbb{R}^{N}}\left[\left|\nabla \phi_{0}\right|^{2}+(1+\lambda g) \phi_{0}^{2}-(1+\alpha) \phi_{0}^{2}\right] d x<0 .
\end{aligned}
$$

So, the conclusion can be proved by taking $e=t \phi_{0}$ with $t>0$ large enough.

(v) By the results of [32] for problem [1.5], there is $\varphi_{\Lambda}>0$ such that

$$
\int_{\mathbb{R}^{N}}\left[\left|\nabla \varphi_{\Lambda}\right|^{2}+\Lambda g(x) \varphi_{\Lambda}^{2}\right] d x=\alpha \int_{\mathbb{R}^{N}} \varphi_{\Lambda}^{2} d x .
$$

Then replacing $\phi_{1}$ by $\varphi_{\Lambda}$ in 2.6) we have

$$
\lim _{t \rightarrow \infty} \frac{I\left(t \varphi_{\Lambda}\right)}{t^{2}} \leq \frac{1}{2}(\lambda-\Lambda) \int_{\mathbb{R}^{N}}\left|\varphi_{\Lambda}\right|^{2} d x<0
$$

since $\lambda \in(\alpha, \Lambda(\alpha))$. Hence, in this case the lemma is proved by taking $e=t \varphi_{\Lambda}$ for $t>0$ large.

\section{Existence of Cerami sequence and its boundedness}

By Lemmas 2.1 and 2.2, we see that the functional $I$ satisfies all the conditions of Proposition 1.1 with $E=H^{\mathrm{I}}\left(\mathbb{R}^{N}\right)$. Thus we have

Lemma 3.1. Under the assumptions of Theorem 1.1 there exists a Cerami sequence $\left\{u_{n}\right\} \subset H^{1}\left(\mathbb{R}^{N}\right)$ such that

$$
I\left(u_{n}\right) \stackrel{n}{\rightarrow} c>0 \quad \text { and } \quad\left\|I^{\prime}\left(u_{n}\right)\right\|_{H^{-1}\left(\mathbb{R}^{N}\right)}\left(1+\left\|u_{n}\right\|\right) \stackrel{n}{\rightarrow} 0,
$$

where $c$ is defined as in Proposition 1.1

Now, the main aim of this section is to show that $\left\{u_{n}\right\}$ obtained in Lemma3.1 is bounded. 
Lemma 3.2. Under the assumptions of Theorem 1.1 the sequence $\left\{u_{n}\right\}$ obtained in Lemma 3.1 is bounded in $H^{1}\left(\mathbb{R}^{N}\right)$.

Proof. For $\beta>0$ (which will be determined below), let

$$
w_{n}=\frac{\beta u_{n}}{\left\|u_{n}\right\|}=\beta_{n} u_{n} \quad \text { with } \quad \beta_{n}=\frac{\beta}{\left\|u_{n}\right\|} .
$$

Clearly, $\left\{w_{n}\right\}$ is bounded in $H^{1}\left(\mathbb{R}^{N}\right)$. Passing to a subsequence and by Lemma 1.1 we may assume that $\left\{w_{n}\right\}$ satisfies the following alternative:

- Vanishing: $\lim _{n \rightarrow \infty} \sup _{y \in \mathbb{R}^{N}} \int_{B_{R}(y)} w_{n}^{2} d x=0$ for all $R>0$.

- Nonvanishing: There exist $v>0, R<\infty$ and $\left\{y_{n}\right\} \subset \mathbb{R}^{N}$ such that

$$
\lim _{n \rightarrow \infty} \int_{B_{R}\left(y_{n}\right)} w_{n}^{2} d x \geq v>0 .
$$

In what follows, we show that if $\left\{u_{n}\right\}$ is not bounded in $H^{1}\left(\mathbb{R}^{N}\right)$, say $\left\|u_{n}\right\| \stackrel{n}{\rightarrow} \infty$, then $\left\{w_{n}\right\}$ satisfies neither Vanishing nor Nonvanishing, a contradiction. Thus the proof of this lemma will be complete.

Claim 1. Vanishing cannot occur.

By 2.5, , for any $\epsilon>0$, there exist $s \in\left(1, \frac{N-2}{N+2}\right)$ and $C_{\epsilon}>0$ such that

$$
\left|\int_{\mathbb{R}^{N}} F\left(w_{n}\right) d x\right| \leq \epsilon \beta^{2}\left|w_{n}\right|_{2}^{2}+C_{\epsilon}\left\|w_{n}\right\|_{s+1}^{s+1} .
$$

By the well-known vanishing lemma [20, Lemma I.1 of part 2], we see that

$$
\int_{\mathbb{R}^{N}} F\left(w_{n}\right) d x \rightarrow 0 \quad \text { as } n \rightarrow \infty .
$$

Hence,

$$
I\left(w_{n}\right)=I\left(\frac{\beta}{\left\|u_{n}\right\|} u_{n}\right)=I\left(\beta_{n} u_{n}\right) \geq \frac{1}{2} \beta^{2}+o(1) ;
$$

here, and in what follows, $o(1)$ denotes a quantity which tends to zero as $n \rightarrow \infty$. Now, we prove Claim 1 under condition $(1.2)$ or 1.3$)$.

If we assume (1.2), from (3.1) and $w_{n}=\beta_{n} u_{n}$, we see that

$$
\begin{aligned}
I\left(w_{n}\right)+o(1) & =I\left(\beta_{n} u_{n}\right)-\frac{\beta_{n}^{2}}{2}\left\langle I^{\prime}\left(u_{n}\right), u_{n}\right\rangle=\int_{\mathbb{R}^{N}}\left[\frac{\beta_{n}^{2}}{2} f\left(u_{n}\right) u_{n}-F\left(\beta_{n} u_{n}\right)\right] d x \\
& \leq D \int_{\mathbb{R}^{N}}\left[\frac{1}{2} f\left(u_{n}\right) u_{n}-F\left(u_{n}\right)\right] d x \quad \text { by }[1.2, \text { for some } D \geq 1 \\
& =D\left(I\left(u_{n}\right)-\frac{1}{2}\left\langle I^{\prime}\left(u_{n}\right), u_{n}\right\rangle\right) \stackrel{n}{\rightarrow} D c \quad \text { by 3.1. }
\end{aligned}
$$


Taking $\beta^{2}>2 D c$, we see that the above inequality contradicts 3.3 and Claim 1 is proved.

Now assume [1.3. As in [16], since $\left\|u_{n}\right\| \stackrel{n}{\rightarrow} \infty$, we have $\beta_{n}=\beta /\left\|u_{n}\right\| \in(0,1)$ for sufficiently large $n$ and it follows from 3.3 that

$$
\max _{t \in[0,1]} I\left(t u_{n}\right) \geq I\left(\frac{\beta}{\left\|u_{n}\right\|} u_{n}\right) \geq \frac{1}{4} \beta^{2} \quad \text { for } n \text { large enough. }
$$

By 3.1,$I\left(u_{n}\right) \stackrel{n}{\rightarrow} c>0$, so there is $M>0$ such that $I\left(u_{n}\right) \leq M$. Taking $\beta>0$ so large that $M<\frac{1}{4} \beta^{2}$, we see that the maximum in the above inequality cannot be attained at $t=0$ or 1 . Thus, for each sufficiently large $n$, there exists $t_{n} \in(0,1)$ such that $I\left(t_{n} u_{n}\right)=\max _{t \in[0,1]} I\left(t u_{n}\right)$ and it follows that $\left\langle I^{\prime}\left(t_{n} u_{n}\right), t_{n} u_{n}\right\rangle=0$. Hence, by 1.3$]$ we have

$$
\begin{aligned}
I\left(t_{n} u_{n}\right) & =I\left(t_{n} u_{n}\right)-\frac{1}{2}\left\langle I^{\prime}\left(t_{n} u_{n}\right), t_{n} u_{n}\right\rangle=\int_{\mathbb{R}^{N}}\left[\frac{1}{2} f\left(t_{n} u_{n}\right) t_{n} u_{n}-F\left(t_{n} u_{n}\right)\right] d x \\
& \leq D \int_{\mathbb{R}^{N}}\left[\frac{1}{2} f\left(u_{n}\right) u_{n}-F\left(u_{n}\right)\right] d x=D\left[I\left(u_{n}\right)-\frac{1}{2}\left\langle I^{\prime}\left(u_{n}\right), u_{n}\right\rangle\right] \\
& \stackrel{n}{\rightarrow} D c .
\end{aligned}
$$

This contradicts 3.4 if we choose further $\beta^{2}>\max \{4 M, 4 D c\}$. So, Vanishing cannot occur.

Claim 2. If Nonvanishing occurs, then $\left\{y_{n}\right\}$ must be bounded.

If $\left\{y_{n}\right\}$ is unbounded, passing to a subsequence we may assume that $\left|y_{n}\right| \stackrel{n}{\rightarrow} \infty$. For any $\varphi \in C_{0}^{\infty}\left(\mathbb{R}^{N}\right)$, setting $\varphi_{n}(x)=\varphi\left(x-y_{n}\right)$, it follows from 3.1 that

$$
\left|\left\langle I^{\prime}\left(u_{n}\right), \varphi_{n}\right\rangle\right| \leq\left\|I^{\prime}\left(u_{n}\right)\right\|_{H^{-1}\left(\mathbb{R}^{N}\right)}\left\|\varphi_{n}\right\|=\left\|I^{\prime}\left(u_{n}\right)\right\|_{H^{-1}\left(\mathbb{R}^{N}\right)}\|\varphi\| \stackrel{n}{\rightarrow} 0 .
$$

Let $\tilde{u}_{n}(x)=u_{n}\left(x+y_{n}\right)$ and $\tilde{w}_{n}(x)=w_{n}\left(x+y_{n}\right)$, where $w_{n}$ is defined by 3.2. Then

$$
\int_{\mathbb{R}^{N}}\left[\nabla \tilde{w}_{n} \nabla \varphi+\left(1+\lambda g\left(x+y_{n}\right)\right) \tilde{w}_{n} \varphi-\frac{f\left(\tilde{u}_{n}\right)}{\tilde{u}_{n}} \tilde{w}_{n} \varphi\right] d x=o(1) .
$$

Since $\left\|w_{n}\right\|=\beta$ and $\left\{w_{n}\right\}$ has the Nonvanishing property, it is not difficult to see that there exists $\tilde{w} \in H^{1}\left(\mathbb{R}^{N}\right) \backslash\{0\}$ such that

$$
\tilde{w}_{n} \stackrel{n}{\rightarrow} \tilde{w} \quad \text { weakly in } H^{1}\left(\mathbb{R}^{N}\right), \quad \tilde{w}_{n} \stackrel{n}{\rightarrow} \tilde{w} \quad \text { a.e. in } \mathbb{R}^{N} .
$$

As $g \in L^{\infty}\left(\mathbb{R}^{N}\right), g\left(x+y_{n}\right) \tilde{w}_{n}$ is bounded in $L^{2}\left(\mathbb{R}^{N}\right)$. Since $g(x) \rightarrow 1$ as $|x| \rightarrow \infty$, and $\left|y_{n}\right| \stackrel{n}{\rightarrow} \infty$, we have

$$
g\left(x+y_{n}\right) \tilde{w}_{n}(x) \stackrel{n}{\rightarrow} \tilde{w}(x) \quad \text { a.e. in } \mathbb{R}^{N}
$$


Hence, $g\left(x+y_{n}\right) \tilde{w}_{n} \stackrel{n}{\rightarrow} \tilde{w}$ weakly in $L^{2}\left(\mathbb{R}^{N}\right)$, that is,

$$
\int_{\mathbb{R}^{N}} g\left(x+y_{n}\right) \tilde{w}_{n} \varphi d x \stackrel{n}{\rightarrow} \int_{\mathbb{R}^{N}} \tilde{w} \varphi d x
$$

Moreover, we claim that

$$
\int_{\mathbb{R}^{N}} \frac{f\left(\tilde{u}_{n}\right)}{\tilde{u}_{n}} \tilde{w}_{n} \varphi d x \stackrel{n}{\rightarrow}(1+\alpha) \int_{\mathbb{R}^{N}} \tilde{w} \varphi d x .
$$

In fact, by 3.1 we see that

$$
\frac{1}{\left\|\tilde{u}_{n}\right\|}\left\langle I^{\prime}\left(u_{n}\right), \tilde{w}_{n}^{-}\right\rangle=o(1)
$$

where $\tilde{w}_{n}^{-}(x)=\max \left\{-\tilde{w}_{n}(x), 0\right\}$, that is,

$$
\int_{\mathbb{R}^{N}}\left[\left|\nabla \tilde{w}_{n}^{-}\right|^{2}+(1+\lambda g(x))\left|\tilde{w}_{n}^{-}\right|^{2}-\frac{f\left(\tilde{u}_{n}\right)}{\tilde{u}_{n}}\left|\tilde{w}_{n}^{-}\right|^{2}\right] d x=o(1) .
$$

Noting that $f(t) \equiv 0$ for all $t<0$ by $(\mathrm{F} 1)$, this yields $\left\|\tilde{w}_{n}^{-}\right\| \stackrel{n}{\rightarrow} 0$ and $\tilde{w} \geq 0$ a.e. in $\mathbb{R}^{N}$. Set

$$
\Omega_{0}=\left\{x \in \mathbb{R}^{N}: \tilde{w}(x)=0\right\}, \quad \Omega_{+}=\left\{x \in \mathbb{R}^{N}: \tilde{w}(x)>0\right\} .
$$

For $x \in \Omega_{0}$, by (F1), (F2) there exists $C>0$ such that $f(t) / t \leq C$ for all $t \in \mathbb{R}$. Then it follows from $\tilde{w}_{n}(x) \stackrel{\text { a.e. }}{\longrightarrow} w(x) \equiv 0$ that

$$
\frac{f\left(\tilde{u}_{n}(x)\right)}{\tilde{u}_{n}(x)} \tilde{w}_{n}(x) \stackrel{n}{\rightarrow} 0 \equiv(1+\alpha) w(x) \quad \text { a.e. in } \Omega_{0} .
$$

For $x \in \Omega_{+}$, since $\left\|\tilde{u}_{n}\right\|=\left\|u_{n}\right\| \stackrel{n}{\rightarrow} \infty$ and $\tilde{w}_{n}(x) \stackrel{n}{\rightarrow} w(x)>0$ a.e. in $\Omega_{+}$, we see that $\tilde{u}_{n}(x) \stackrel{n}{\rightarrow} \infty$. Then $(\mathrm{F} 2)$ yields

$$
\frac{f\left(\tilde{u}_{n}(x)\right)}{\tilde{u}_{n}(x)} \stackrel{n}{\rightarrow} 1+\alpha \quad \text { a.e. in } \Omega_{2} .
$$

Hence,

$$
\frac{f\left(\tilde{u}_{n}(x)\right)}{\tilde{u}_{n}(x)} \tilde{w}_{n}(x) \stackrel{n}{\rightarrow}(1+\alpha) w(x) \quad \text { a.e. in } \mathbb{R}^{N} .
$$

Since $f(t) / t \leq C$ for all $t \in \mathbb{R}$, we see that $\left\{\frac{f\left(\tilde{u}_{n}(x)\right)}{\tilde{u}_{n}(x)} \tilde{w}_{n}(x)\right\}$ is bounded in $L^{2}\left(\mathbb{R}^{N}\right)$. Therefore,

$$
\frac{f\left(\tilde{u}_{n}(x)\right)}{\tilde{u}_{n}(x)} \tilde{w}_{n}(x) \stackrel{n}{\rightarrow}(1+\alpha) w(x) \quad \text { weakly in } L^{2}\left(\mathbb{R}^{N}\right) .
$$

This implies (3.7).

Using 3.5 - 3.7, we see that $\tilde{w}$ satisfies

$$
-\Delta \tilde{w}+(1+\lambda) \tilde{w}=(1+\alpha) \tilde{w} \quad \text { in } H^{1}\left(\mathbb{R}^{N}\right) .
$$


This is impossible since the above equation has only the zero solution in $H^{1}\left(\mathbb{R}^{N}\right)$ for any $\alpha$ and $\lambda$ by the Pohozaev identity [4], a contradiction.

Claim 3. Nonvanishing cannot occur.

By Claim 2, if $\left\{w_{n}\right\}$ has the nonvanishing property, then $\left\{y_{n}\right\}$ is bounded, and there exists $0 \not \equiv w \in H^{1}\left(\mathbb{R}^{N}\right)$ such that

$$
w_{n} \stackrel{n}{\rightarrow} w \quad \text { weakly in } H^{1}\left(\mathbb{R}^{N}\right) .
$$

By our assumption $\left\|u_{n}\right\| \stackrel{n}{\rightarrow} \infty$, so noting 3 3.1, we have $\left\|I^{\prime}\left(u_{n}\right)\right\|_{H^{-1}\left(\mathbb{R}^{N}\right)}\left\|u_{n}\right\| \stackrel{n}{\rightarrow} 0$, and thus

$$
\frac{1}{\left\|u_{n}\right\|^{2}}\left\langle I^{\prime}\left(u_{n}\right), u_{n}^{-}\right\rangle=o(1)
$$

where $u_{n}^{-}(x)=\max \left\{-u_{n}(x), 0\right\}$, that is,

$$
\int_{\mathbb{R}^{N}}\left[\left|\nabla w_{n}^{-}\right|^{2}+(1+\lambda g(x))\left|w_{n}^{-}\right|^{2}-\frac{f\left(u_{n}\right)}{u_{n}}\left|w_{n}^{-}\right|^{2}\right] d x=o(1) .
$$

This implies that $\left\|w_{n}^{-}\right\| \stackrel{n}{\rightarrow} 0$ since $f(t) \equiv 0$ for all $t<0$ by (F1). So $w \geq 0$ a.e. on $\mathbb{R}^{N}$.

For any $\varphi \in C_{0}^{\infty}\left(\mathbb{R}^{N}\right)$, we have $\frac{1}{\left\|u_{n}\right\|}\left\langle I^{\prime}\left(u_{n}\right), \varphi\right\rangle=o(1)$, that is,

$$
\int_{\mathbb{R}^{N}}\left[\nabla w_{n} \nabla \varphi+V_{\lambda}(x) w_{n} \varphi\right] d x-\int_{\mathbb{R}^{N}} \frac{f\left(u_{n}\right)}{u_{n}} w_{n} \varphi d x=o(1) .
$$

Since $w_{n} \stackrel{n}{\rightarrow} w$ weakly in $H^{1}\left(\mathbb{R}^{N}\right)$, we get

$$
\int_{\mathbb{R}^{N}}\left[\nabla w_{n} \nabla \varphi+V_{\lambda}(x) w_{n} \varphi\right] d x \stackrel{n}{\rightarrow} \int_{\mathbb{R}^{N}}\left[\nabla w \nabla \varphi+V_{\lambda}(x) w \varphi\right] d x .
$$

Similar to 3.77, we have

$$
\int_{\mathbb{R}^{N}} \frac{f\left(u_{n}\right)}{u_{n}} w_{n} \varphi d x \stackrel{n}{\rightarrow}(1+\alpha) \int_{\mathbb{R}^{N}} w \varphi d x .
$$

From $3.8-3.10$ we see that $w \geq 0$ and satisfies

$$
-\Delta w+(1+\lambda g(x)) w=(1+\alpha) w .
$$

Now, we show that for all $\lambda$ and $\alpha$ in cases (i) to (v) of Theorem 1.1, there is always a contradiction to 3.11. Hence, Claim 3 is proved.

Case (i). Since $\alpha>0$ and $0<\lambda<\alpha$, we have

$$
1+\alpha>1+\lambda \geq \inf \sigma\left(-\Delta+V_{\lambda}(x)\right)
$$

and Proposition 2.1 (ii) implies that 3.11) is impossible. 
Case (ii). For $\alpha>\Gamma$ and $\lambda=\alpha$, there exists $v \in H^{1}\left(\mathbb{R}^{N}\right) \backslash\{0\}$ such that

$$
\int_{\mathbb{R}^{N}}|\nabla v|^{2} d x<\alpha \int_{\mathbb{R}^{N}}(1-g(x)) v^{2} d x,
$$

that is,

$$
1+\alpha>\frac{\int_{\mathbb{R}^{N}}\left[|\nabla v|^{2}+(1+\alpha g(x)) v^{2}\right] d x}{\int_{\mathbb{R}^{N}} v^{2} d x},
$$

which implies that

$$
1+\alpha>\inf \sigma\left(-\Delta+V_{\alpha}(x)\right) .
$$

So, (3.11) is also impossible by Proposition 2.1.ii).

Case (iii). Since $\alpha>\xi_{1}>0$, if $0<\lambda<\alpha$, this case has been discussed in Case (i). For $\alpha>\xi_{1}$ with $\alpha \leq \lambda<\infty$, it follows from Proposition 2.2 i) that 3.11 is impossible.

Case (iv). For $\alpha=\xi_{1}$ and $\lambda>\alpha$, Proposition 2.2 (i) also shows that 3.11) is impossible.

Case (v). In this case, 3.11) is impossible by Proposition 2.2.ii).

\section{Proofs of the main results}

The aim of this section is to prove Theorems 1.1 to 1.3 . In order to show Theorem 1.1, we introduce the following problem:

$$
\left\{\begin{array}{l}
-\Delta u+(1+\lambda) u=f(u) \quad \text { in } \mathbb{R}^{N} \\
u \in H^{1}\left(\mathbb{R}^{N}\right), \quad N \geq 3
\end{array}\right.
$$

which is usually called the limit problem associated to 1.1 at infinity. Its energy functional is defined by

$$
I^{\infty}(u)=\frac{1}{2} \int_{\mathbb{R}^{N}}\left[|\nabla u|^{2}+(1+\lambda) u^{2}\right] d x-\int_{\mathbb{R}^{N}} F(u) d x, \quad F(u)=\int_{0}^{u} f(s) d s .
$$

Under the conditions of Theorem 1.1. Lemma 3.2 implies that there exists a bounded Cerami sequence $\left\{u_{n}\right\}$ such that 3.1 holds. Then the usual way to prove Theorem 1.1 is to show that the functional $I$ satisfies the so called Cerami condition (or PS condition), that is, $\left\{u_{n}\right\}$ has a subsequence which converges strongly in $H^{1}\left(\mathbb{R}^{N}\right)$. However, this is usually complicated or difficult. Motivated by [16], instead of verifying the Cerami condition for $I$, we prove Theorem 1.1 by directly showing that $\left\{u_{n}\right\}$ converges weakly to a nontrivial solution of (1.1), although $I$ does satisfy the Cerami condition except in case (ii) of Theorem 1.1 (see our Proposition 4.1 below).

Lemma 4.1. Suppose that (F1), (F2), (G1) hold and

$$
F(s) \leq \frac{1}{2} f(s) s \quad \text { for all } s>0 .
$$

Moreover, let $\left\{u_{n}\right\} \subset H^{1}\left(\mathbb{R}^{N}\right)$ be a bounded Cerami sequence for I at level $c>0$, that is, (3.1) holds. Then there is a subsequence of $\left\{u_{n}\right\}$, still denoted by $\left\{u_{n}\right\}$, such that $u_{n} \stackrel{n}{\rightarrow} u \not \equiv 0$ weakly in $H^{1}\left(\mathbb{R}^{N}\right)$ and $I^{\prime}(u)=0$. 
Proof. Since $\left\{u_{n}\right\}$ is bounded, there exists a subsequence, still denoted by $\left\{u_{n}\right\}$, such that $u_{n} \stackrel{n}{\rightarrow} u$ weakly in $H^{1}\left(\mathbb{R}^{N}\right)$, but $u$ may be identically equal to 0 . Since $\left\|I^{\prime}\left(u_{n}\right)\right\|_{H^{-1}\left(\mathbb{R}^{N}\right)}$ $\stackrel{n}{\rightarrow} 0$, it is easy to see that $I^{\prime}(u)=0$ and

$$
\left\langle I^{\prime}\left(u_{n}\right)-I^{\prime}(u), \varphi\right\rangle \stackrel{n}{\rightarrow} 0 \quad \text { for any } \varphi \in C_{0}^{\infty}\left(\mathbb{R}^{N}\right) .
$$

The main point is to prove that $u \neq \equiv 0$. By contradiction, if $u \equiv 0$, we claim that $\left\{u_{n}\right\}$ is also a (PS) sequence for $I^{\infty}$ at level $c$. In fact, since $g(x) \rightarrow 1$ as $|x| \rightarrow \infty$, and $u_{n} \stackrel{n}{\rightarrow} 0$ strongly in $L_{\text {loc }}^{2}\left(\mathbb{R}^{N}\right)$, we see that

$$
I^{\infty}\left(u_{n}\right)-I\left(u_{n}\right)=\lambda \int_{\mathbb{R}^{N}}(1-g(x)) u_{n}^{2} d x \stackrel{n}{\rightarrow} 0
$$

and

$$
\sup _{\|\varphi\| \leq 1}\left|\left\langle I^{\infty \prime}\left(u_{n}\right)-I^{\prime}\left(u_{n}\right), \varphi\right\rangle\right|=\lambda \sup _{\|\varphi\| \leq 1}\left|\int_{\mathbb{R}^{N}}(1-g(x)) u_{n} \varphi d x\right| \stackrel{n}{\rightarrow} 0 .
$$

Next, we claim that $\left\{u_{n}\right\}$ does not vanish. Otherwise, by (F1), (F2) and the vanishing lemma [20], we get

$$
\int_{\mathbb{R}^{N}} f\left(u_{n}\right) u_{n} d x \stackrel{n}{\rightarrow} 0 .
$$

Then $\left\langle I^{\prime}\left(u_{n}\right), u_{n}\right\rangle \stackrel{n}{\rightarrow} 0$ and 4.4 imply that $\left\|u_{n}\right\| \stackrel{n}{\rightarrow} 0$. This is impossible since $I\left(u_{n}\right) \stackrel{n}{\rightarrow}$ $c>0$.

So, $\left\{u_{n}\right\}$ is nonvanishing. Let $\tilde{u}_{n}(x)=u_{n}\left(x+y_{n}\right)$. Similar to the proof of Claim 2 of Lemma 3.2, we find that, up to a subsequence, $\tilde{u}_{n} \stackrel{n}{\rightarrow} \tilde{u} \not \equiv 0$ weakly in $H^{1}\left(\mathbb{R}^{N}\right)$ with $I^{\infty^{\prime}}(\tilde{u})=0$ and $\tilde{u}_{n} \stackrel{n}{\rightarrow} \tilde{u}$ a.e. in $\mathbb{R}^{N}$.

By $(\mathrm{F} 1)$ and 4.3 we have $\frac{1}{2} f(s) s-F(s) \geq 0$ for all $s \in \mathbb{R}$. From Fatou's lemma we get

$$
\begin{aligned}
c & =\lim _{n \rightarrow \infty}\left[I^{\infty}\left(u_{n}\right)-\frac{1}{2}\left\langle I^{\infty^{\prime}}\left(u_{n}\right), u_{n}\right\rangle\right]=\lim _{n \rightarrow \infty}\left[I^{\infty}\left(\tilde{u}_{n}\right)-\frac{1}{2}\left\langle I^{\infty^{\prime}}\left(\tilde{u}_{n}\right), \tilde{u}_{n}\right\rangle\right] \\
& =\lim _{n \rightarrow \infty} \int_{\mathbb{R}^{N}}\left[\frac{1}{2} f\left(\tilde{u}_{n}\right) \tilde{u}_{n}-F\left(\tilde{u}_{n}\right)\right] d x \geq \int_{\mathbb{R}^{N}}\left[\frac{1}{2} f(\tilde{u}) \tilde{u}-F(\tilde{u})\right] d x \\
& =I^{\infty}(\tilde{u})-\frac{1}{2}\left\langle I^{\infty \prime}(\tilde{u}), \tilde{u}\right\rangle=I^{\infty}(\tilde{u}) .
\end{aligned}
$$

Therefore, $\tilde{u}$ is a nontrivial critical point of $I^{\infty}$ satisfying $I^{\infty}(\tilde{u}) \leq c$, and the strong maximum principle implies that $\tilde{u}>0$ on $\mathbb{R}^{N}$. Furthermore, $\tilde{u}$ satisfies the so called Pohozaev-type identity [4],

$$
\frac{2 N}{N-2} \int_{\mathbb{R}^{N}}|\nabla \tilde{u}|^{2} d x=\frac{-1}{2}(1+\lambda) \int_{\mathbb{R}^{N}} \tilde{u}^{2} d x+\int_{\mathbb{R}^{N}} F(\tilde{u}) d x .
$$

Similar to [18] or [16], we set $v_{t}(x)=\tilde{u}(x / t)$ for $t>k 0$. Then by direct calculation and (4.5), we have

$$
I^{\infty}\left(v_{t}\right)=\left(\frac{1}{2} t^{N-2}-\frac{N-2}{2 N} t^{N}\right)|\nabla \tilde{u}|_{2}^{2}
$$


Since $N \geq 3$, there exists $t_{0}>0$ large enough such that $I^{\infty}\left(v_{t}\right)<0$ for all $t \geq t_{0}$ and

$$
\sup _{t \geq 0} I^{\infty}\left(v_{t}\right)=I^{\infty}\left(v_{1}\right)=I^{\infty}(\tilde{u}), \quad \text { where } \quad v_{1}=\left.v_{t}(x)\right|_{t=1}=\tilde{u} .
$$

Let $\gamma(t)(x)=\tilde{u}\left(x / t t_{0}\right)$ for $0<t \leq 1$ and $\gamma(t)(x) \equiv 0$ for $t=0$. Then $\gamma \in$ $C\left([0,1], H^{1}\left(\mathbb{R}^{N}\right)\right)$ and $I^{\infty}(\gamma(1))<0$. Hence by the definition of $c$ and the continuity of $I$, there is $t^{*} \in(0,1]$ such that

$$
c \leq \max _{t \in[0,1]} I(\gamma(t))=I\left(\gamma\left(t^{*}\right)\right)<I^{\infty}\left(\gamma\left(t^{*}\right)\right) \leq \sup _{t>0} I^{\infty}\left(v_{t}\right)=I^{\infty}(\tilde{u}) \leq c ;
$$

here 4.7) is used and $I\left(\gamma\left(t^{*}\right)\right)<I^{\infty}\left(\gamma\left(t^{*}\right)\right)$ is obtained by (G1). This is a contradiction.

Proof of Theorem 1.1. By Lemma 3.2, we see easily that Theorem 1.1 is a straightforward consequence of Lemma 4.1

Proof of Theorem 1.2. Since $\lambda=0$, problem (1.1) or (4.1) becomes

$$
-\Delta u+u=f(u), \quad u \in H^{1}\left(\mathbb{R}^{N}\right) .
$$

Similar to [16], define $h(s)=-s+f(s)$ if $s \geq 0$ and $h(s)=-h(-s)$ for $s<0$. Then, by (F1), (F2) with $\alpha>0$, we see that $h$ satisfies all the conditions of Lions [4], that is, $h$ : $\mathbb{R} \rightarrow \mathbb{R}$ is continuous and odd, $\lim _{s \rightarrow 0^{+}} h(s) / s=-1<0, \lim _{s \rightarrow \infty}|h(s)| / s^{(N+2) /(N-2)}$ $=0$, and there exists $s_{0}>0$ such that $H\left(s_{0}\right)>0$, where $H(s)=\int_{0}^{s} h(t) d t$. Hence, by [4] there is a positive least energy solution $w \in H^{1}\left(\mathbb{R}^{N}\right)$ for

$$
-\Delta u=h(u), \quad u \in H^{1}\left(\mathbb{R}^{N}\right) .
$$

By the definition of $h$, we see that $w$ is essentially a positive solution of problem 1.1 with $\lambda=0$.

Proof of Theorem 1.3. By (F1)-(F3), since $\alpha=0$,

$$
\int_{\mathbb{R}^{N}} f(u) u d x \leq \int_{\mathbb{R}^{N}} u^{2} d x, \quad \forall u \in H^{1}\left(\mathbb{R}^{N}\right) .
$$

So, problem 1.1 has no positive solution for any $\lambda \geq 0$.

Before ending this section, we mention that under the assumptions of Theorem 1.1 , the energy functional $I$ does satisfy the Cerami condition except in case (ii). We have the following proposition.

Proposition 4.1. Under the assumptions of Theorem 1.1 the Cerami sequence $\left\{u_{n}\right\} \subset$ $H^{1}\left(\mathbb{R}^{N}\right)$ obtained in Lemma 3.1 contains a convergent subsequence for cases (i) and (iii)-(v) of Theorem 1.1

To prove this proposition, the following lemma is crucial. 
Lemma 4.2. Suppose that (F1), (G1) hold and

$$
f(s) \leq(1+\alpha) s \quad \text { for all } s>0 .
$$

Let $\lambda \in(\alpha, \infty)$ and $\left\{u_{n}\right\} \subset H^{1}\left(\mathbb{R}^{N}\right)$ be a bounded Cerami sequence for I at level $c>0$. Then for any $\epsilon>0$, there exist $R(\epsilon)$ and $n(\epsilon)>0$ such that

$$
\int_{|x| \geq R}\left[\left|\nabla u_{n}\right|^{2}+\left|u_{n}\right|^{2}\right] d x \leq \epsilon \quad \text { for all } R \geq R(\epsilon) \text { and } n \geq n(\epsilon) .
$$

Proof. The proof is motivated by [9]. For any fixed $R>0$, we take a cut-off function $\xi_{R} \in C^{\infty}\left(\mathbb{R}^{N}, \mathbb{R}\right)$ such that

$$
\xi_{R}(x)= \begin{cases}0, & |x| \leq R / 2 \\ 1, & |x| \geq R\end{cases}
$$

and there exists $C_{0}>0$ (independent of $R$ ) such that $\left|\nabla \xi_{R}(x)\right| \leq C_{0} / R$ for all $x \in \mathbb{R}^{N}$. Then, for any $u \in H^{1}\left(\mathbb{R}^{N}\right)$ and all $R \geq 1$, there exists $C_{1}>0$ (independent of $R$ ) such that $\left\|\xi_{R} u\right\| \leq C_{1}\|u\|$.

Since $\left\{u_{n}\right\} \subset H^{1}\left(\mathbb{R}^{N}\right)$ is a Cerami sequence, for $n$ large we have

$$
\left\langle I^{\prime}\left(u_{n}\right), \xi_{R} u_{n}\right\rangle \leq\left\|I^{\prime}\left(u_{n}\right)\right\|_{H^{-1}\left(\mathbb{R}^{N}\right)}\left\|\xi_{R} u_{n}\right\| \leq o(1),
$$

that is,

$$
\begin{aligned}
\int_{\mathbb{R}^{N}}\left(\left|\nabla u_{n}\right|^{2}+V_{\lambda}(x) u_{n}^{2}\right) \xi_{R} d x+\int_{\mathbb{R}^{N}} u_{n} \nabla u_{n} \nabla \xi_{R} d x & \\
& \leq \int_{\mathbb{R}^{N}} f\left(u_{n}\right) u_{n} \xi_{R} d x+o(1) .
\end{aligned}
$$

Since $\lambda>\alpha$, choosing $\delta=(\lambda-\alpha) / 2>0$, by condition (G1) there exists $R_{1}>0$ such that $V_{\lambda}(x) \geq 1+\alpha+\delta$ for $|x| \geq R_{1}$. From (F1) and 4.9) we have $f\left(u_{n}\right) u_{n} \leq(1+\alpha) u_{n}^{2}$. Then, for $R \geq 2 R_{1}$, 4.12) yields

$$
\int_{\mathbb{R}^{N}}\left(\left|\nabla u_{n}\right|^{2}+\delta u_{n}^{2}\right) \xi_{R} d x \leq\left|\int_{\mathbb{R}^{N}} u_{n} \nabla u_{n} \nabla \xi_{R} d x\right|+o(1) \leq \frac{C_{0}}{R}\left\|u_{n}\right\|^{2}+o(1) .
$$

Since $\left\{u_{n}\right\} \subset H^{1}\left(\mathbb{R}^{N}\right)$ is bounded, taking $R$ and $n$ large enough in 4.13 we see that 4.10) holds.

Finally, we give the proof of Proposition 4.1

Proof of Proposition 4.1. Case (i). Let $\left\{u_{n}\right\}$ be given by Lemma 3.1, that is,

$$
I\left(u_{n}\right) \stackrel{n}{\rightarrow} c>0 \quad \text { and } \quad\left\|I^{\prime}\left(u_{n}\right)\right\|_{H^{-1}\left(\mathbb{R}^{N}\right)} \stackrel{n}{\rightarrow} 0,
$$

where

$$
c=\inf _{\gamma \in \Gamma} \max _{0 \leq t \leq 1} I(\gamma(t)), \quad \Gamma=\left\{\gamma \in C\left([0,1], H^{1}\left(\mathbb{R}^{N}\right)\right): \gamma(0)=0, I(\gamma(1))<0\right\} .
$$


From Lemma 3.2, we know that $\left\{u_{n}\right\}$ is bounded in $H^{1}\left(\mathbb{R}^{N}\right)$. By the proof of Theorem 1.2 (see Steps 2 and 3 ) in [18], to prove this proposition we need only show that, passing to a subsequence, $\left\{u_{n}\right\}$ is tight, that is, for any $\epsilon>0$, there exists $R>0$ such that

$$
\int_{|x| \geq R} \mid\left[\left.\nabla u_{n}\right|^{2}+u_{n}^{2}\right] d x<\epsilon
$$

Following [18], we rewrite [1.1] as

$$
-\Delta u+u=f_{\lambda}(x, u), \quad u \in H^{1}\left(\mathbb{R}^{N}\right),
$$

where $f_{\lambda}(x, u)=-\lambda g(x) u+f(u)$, and set $\bar{f}_{\lambda}(u)=-\lambda u+f(u)$. Using (G1), we see that

$$
\begin{array}{ll}
f_{\lambda}(x, t) \geq \bar{f}_{\lambda}(t) & \text { for any }(x, t) \in \mathbb{R}^{N} \times[0, \infty), \\
f_{\lambda}(x, t)>\bar{f}_{\lambda}(t) & \text { for any }(x, t) \in \Omega \times(0, \infty),
\end{array}
$$

where $\Omega$ is given by $(\mathrm{G} 1)$. If $f_{\lambda}(x, t)$ and $\bar{f}_{\lambda}(t)$ satisfy all the conditions (C1) to (C6) in [18], then the proof of (4.15) is exactly the same as Step 2 of the proof of Theorem 1.2 there. However, it is obvious that $f_{\lambda}(x, t)$ and $\bar{f}_{\lambda}(t)$ do not satisfy all those conditions. So we have to modify part of the proof of [18, Theorem 1.2]. Moreover, with the notations $f_{\lambda}(x, t)$ and $\bar{f}_{\lambda}(t)$, the functionals $I$ and $I^{\infty}$ defined by 1.7$)$ and 4.2$)$ can be written as

$$
\begin{array}{cc}
I(u)=\frac{1}{2}\|u\|^{2}-\int_{\mathbb{R}^{N}} F_{\lambda}(x, u) d x & \text { with } F_{\lambda}(x, t)=\int_{0}^{t} f_{\lambda}(x, s) d s, \\
I^{\infty}(u)=\frac{1}{2}\|u\|^{2}-\int_{\mathbb{R}^{N}} \bar{F}(u) d x & \text { with } \bar{F}_{\lambda}(t)=\int_{0}^{t} \bar{f}_{\lambda}(s) d s .
\end{array}
$$

Clearly, $I(u)<I^{\infty}(u)$ if $u>0$, by 4.17 ).

By Step 2 of the proof of Theorem 1.2 in [18], we know that it is very important to have $c \in\left(0, J^{\infty}\right)$ while proving 4.15 by the classical concentration-compactness principle, where

$$
J^{\infty}=\inf \left\{I^{\infty}(u): u \in \Lambda\right\}
$$

and $\Lambda=\left\{u \in H^{1}\left(\mathbb{R}^{N}\right) \backslash\{0\}:\left\langle I^{\infty \prime}(u), u\right\rangle=0\right\}$.

We claim that $J^{\infty}>0$ and it is attained in $\Lambda$. To prove these facts, some strong restrictions are imposed on $f_{\lambda}(x, t)$ and $\partial_{t} \bar{f}_{\lambda}(t)$ in [18]. Here, we use a different approach and require only our conditions $(\mathrm{F} 1)-(\mathrm{F} 3),(\mathrm{G} 1)$ and $\lambda \in(0, \alpha)$. For this purpose, we define

$$
h(s)= \begin{cases}-(1+\lambda) s+f(s) & \text { if } s \geq 0 \\ -h(-s) & \text { if } s<0\end{cases}
$$

As in the above proof of Theorem 1.2 (where $\lambda=0$ ), by (F1), (F2) and $\lambda \in(0, \alpha)$ we know that this $h(s)$ also satisfies all the conditions in [4]. Note that $\lambda \in(0, \alpha)$ is required to ensure that there exists $s_{0}>0$ such that $H\left(s_{0}\right)>0$. So, by the result of [4], problem 4.1) has a least energy solution $w \in H^{1}\left(\mathbb{R}^{N}\right)$ with $w>0$ on $\mathbb{R}^{N}$ and $J^{\infty}=I^{\infty}(w)$. 
Setting $w_{t}(x)=w(x / t)$ for $t>0$, similar to the proof of (4.6) and (4.7), we see that there exists $t_{0}>0$ large enough such that

$$
I^{\infty}\left(w_{t_{0}}\right)<0 \quad \text { and } \quad \sup _{t \geq 0} I^{\infty}\left(w_{t}\right)=I^{\infty}(w)=J^{\infty}
$$

Then, exactly as for 4.8, we see that $c<J^{\infty}$.

Since $c \in\left(0, J^{\infty}\right)$, we can now follow almost exactly Step 2 of the proof of Theorem 1.2 in [18] to show that 4.15$]$ holds. Here we just keep in mind that the functions $f(x, t)$, $\bar{f}(t), F(x, t)$ and $\bar{F}(t)$ appearing in [18] should be replaced, respectively, by $f_{\lambda}(x, t)$, $\bar{f}_{\lambda}(t), F_{\lambda}(x, t)$ and $\bar{F}_{\lambda}(t)$. The details of the proof are given in the Appendix.

Case (iii). If $\alpha>\xi_{1}$ and $0<\lambda<\alpha$, by Case (i) we see that the Cerami condition holds. If $\alpha>\xi_{1}$ and $\alpha<\lambda<\infty$, it follows from Lemmas 3.2 and 4.2 that the Cerami condition holds.

If $\alpha>\xi_{1}$ and $\lambda=\alpha$, since $\xi_{1}>\Gamma$, this falls under Case (ii). We have no idea how to prove that the Cerami condition holds in this case.

Cases (iv) and (v). These are direct consequences of Lemmas 3.2 and 4.2

\section{The proof of Theorem 1.4}

For fixed $\alpha>0$ and any $0<\lambda<\alpha$, let $u_{\lambda}$ be the positive solution of 1.1 given by Theorem 1.1. We study the asymptotic behavior of $u_{\lambda}$ as $\lambda \rightarrow 0^{+}$.

Lemma 5.1. For fixed $\alpha>0$ and any $\lambda_{0} \in(0, \alpha)$, there exist $b_{1}=b_{1}\left(\alpha, \lambda_{0}\right)$ and $b_{2}=b_{2}(\alpha)>0$ such that

$$
b_{2} \leq I_{\lambda}\left(u_{\lambda}\right) \leq b_{1} \quad \text { for all } \lambda \in\left(0, \lambda_{0}\right]
$$

Here $I_{\lambda}$ denotes the energy functional defined in 1.7 .

Proof. For $\lambda \in(0, \alpha)$, by Proposition 4.1 there is $u_{\lambda} \in H^{1}\left(\mathbb{R}^{N}\right)$ such that

$$
I_{\lambda}\left(u_{\lambda}\right)=\inf _{\gamma \in \Gamma_{\lambda}} \max _{t \in[0,1]} I_{\lambda}(\gamma(t)),
$$

with $\Gamma_{\lambda}=\left\{\gamma \in C\left([0,1], H^{1}\left(\mathbb{R}^{N}\right)\right): \gamma(0)=0\right.$ and $\left.I_{\lambda}(\gamma(1))<0\right\}$. By Lemma 2.1, there exists $b_{2}=b_{2}(\alpha)>0$ such that

$$
I_{\lambda}\left(u_{\lambda}\right) \geq b_{2}, \quad \forall \lambda \in\left(0, \lambda_{0}\right]
$$

On the other hand, since $\lambda_{0}<\alpha$, there exists $\phi \in H^{1}\left(\mathbb{R}^{N}\right)$ with $\phi \geq 0$ such that

$$
\int_{\mathbb{R}^{N}}|\nabla \phi|^{2} d x<\left(\alpha-\lambda_{0}\right) \int_{\mathbb{R}^{N}} \phi^{2} d x .
$$


Similar to the proof of Lemma 2.2(i) and by Fatou's lemma we can prove that there exists $t_{0}=t_{0}\left(\alpha, \lambda_{0}\right)>0$ such that $I_{\lambda_{0}}\left(t_{0} \phi\right)<0$. Thus, $I_{\lambda}\left(t_{0} \phi\right) \leq I_{\lambda_{0}}\left(t_{0} \phi\right)<0$ for all $\lambda \in$ $\left(0, \lambda_{0}\right]$ and

$$
I_{\lambda}\left(u_{\lambda}\right) \leq \max _{t \in[0,1]} I_{\lambda}\left(t t_{0} \phi\right) \leq \max _{t \in[0,1]} I_{\lambda_{0}}\left(t t_{0} \phi\right)=: b_{1} \quad \text { for all } \lambda \in\left(0, \lambda_{0}\right] . \square
$$

Proof of Theorem 1.4. We claim that for fixed $\alpha>0$ and any $\lambda_{0} \in(0, \alpha)$, there exist $C_{1}, C_{2}>0$, depending on $\alpha$ and $\lambda_{0}$, such that

$$
C_{2} \leq\left\|u_{\lambda}\right\| \leq C_{1} \quad \text { for all } \lambda \in\left(0, \lambda_{0}\right] .
$$

In fact, suppose that there exists a sequence $\left\{\lambda_{n}\right\} \subset\left(0, \lambda_{0}\right]$ such that $\left\|u_{\lambda_{n}}\right\| \stackrel{n}{\rightarrow} 0$. Then $I_{\lambda_{n}}\left(u_{\lambda_{n}}\right) \stackrel{n}{\rightarrow} 0$. By Lemma 5.1 this is impossible. Thus $\left\|u_{\lambda}\right\| \geq C_{2}$ for all $\lambda \in\left(0, \lambda_{0}\right]$.

On the other hand, if there exists a sequence $\lambda_{n} \in\left(0, \lambda_{0}\right]$ such that $\left\|u_{\lambda_{n}}\right\| \rightarrow \infty$ as $n \rightarrow \infty$, then setting $u_{n}=u_{\lambda_{n}}$ and $w_{n}=u_{n} /\left\|u_{n}\right\|$, by Lemma 5.1 and similarly to the proof of Lemma 3.2, we get the desired contradiction when Vanishing or Nonvanishing occurs. Hence $\left\|u_{\lambda}\right\| \leq C_{1}$ for all $\lambda \in\left(0, \lambda_{0}\right]$.

If $g(x)$ is radial, we may assume that $u_{\lambda} \in H_{r}^{1}\left(\mathbb{R}^{N}\right)$. Since we get the uniform upper and lower bound for $u_{\lambda}$ as $\lambda \rightarrow 0^{+}$, there exists $u_{0} \in H_{r}^{1}\left(\mathbb{R}^{N}\right)$ such that

$$
u_{\lambda} \stackrel{\lambda}{\rightarrow} u_{0} \quad \text { weakly in } H_{r}^{1}\left(\mathbb{R}^{N}\right) .
$$

By the compactness of the Sobolev embedding $H_{r}^{1}\left(\mathbb{R}^{N}\right) \hookrightarrow L^{p}\left(\mathbb{R}^{N}\right)$ for $2<p<$ $2 N /(N-2)$ (see [27]), and a standard procedure, we see that

$$
u_{\lambda} \stackrel{\lambda}{\rightarrow} u_{0} \not \equiv 0 \quad \text { strongly in } H_{r}^{1}\left(\mathbb{R}^{N}\right),
$$

and $u_{0}$ solves

$$
-\Delta u+u=f(u), \quad u \in H^{1}\left(\mathbb{R}^{N}\right) .
$$

\section{Appendix}

For the sake of completeness, we give here the details of the proof of 4.15 by using the classical concentration-compactness principle.

From Lemma 3.2 $\left\{u_{n}\right\}$ is bounded in $H^{1}\left(\mathbb{R}^{N}\right)$. Passing to a subsequence, we may assume that, for some $\ell \geq 0$,

$$
\int_{\mathbb{R}^{N}}\left|\nabla u_{n}\right|^{2}+u_{n}^{2} d x \stackrel{n}{\rightarrow} \ell .
$$

We claim that $\ell>0$. Otherwise, if $\ell=0$, then $I\left(u_{n}\right) \stackrel{n}{\rightarrow} 0$, which leads to a contradiction by (4.14) and $c>0$. Let

$$
\rho_{n}(x)=\left|\nabla u_{n}\right|^{2}+u_{n}^{2}, \quad Q_{n}(t)=\sup _{y \in \mathbb{R}^{N}} \int_{B_{t}(y)} \rho_{n}(x) d x,
$$

where $B_{t}(y)=\left\{x \in \mathbb{R}^{N}:|x-y| \leq t\right\}$. 
By exactly the same discussion as in [20, Lemma I.1 of part 1], we know that $Q_{n}(t) \stackrel{n}{\rightarrow} Q(t) \geq 0 \quad$ for all $t \geq 0, \quad Q(t)$ is nondecreasing, $\quad Q(t) \stackrel{t \rightarrow \infty}{\longrightarrow} \tau \in[0, \ell]$, and then only one of the following three cases can occur:
(A) $\tau=0$ (vanishing);
(B) $\tau \in(0, \ell)$ (dichotomy);
(C) $\tau=\ell$ (compactness).

Case (A) does not occur. In fact, if $\tau=0$, it follows from (F1), (F2) and the wellknown vanishing lemma of Lions [20, Lemma I.1 of part 2] that

$$
\lim _{n \rightarrow \infty} \int_{\mathbb{R}^{N}} f\left(u_{n}\right) u_{n} d x=\lim _{n \rightarrow \infty} \int_{\mathbb{R}^{N}} F\left(u_{n}\right) d x=0 .
$$

By $4.14,,\left\langle I^{\prime}\left(u_{n}\right), u_{n}\right\rangle \stackrel{n}{\rightarrow} 0$, so

$$
\begin{aligned}
I\left(u_{n}\right) & =\frac{1}{2}\left\|u_{n}\right\|^{2}-\int_{\mathbb{R}^{N}} F_{\lambda}\left(x, u_{n}\right) d x=\int_{\mathbb{R}^{N}}\left[\frac{1}{2} f_{\lambda}\left(x, u_{n}\right)-F_{\lambda}\left(x, u_{n}\right)\right] d x+o(1) \\
& =\int_{\mathbb{R}^{N}}\left[\frac{1}{2} f\left(u_{n}\right) u_{n}-F\left(u_{n}\right)\right] d x \stackrel{n}{\rightarrow} 0 .
\end{aligned}
$$

This is impossible by (4.14) and $c>0$.

Next, we exclude Case (B) by using the fact that $c \in\left(0, J^{\infty}\right)$.

If Case (B) occurs, that is, $\tau \in(0, \ell)$, from $Q(t) \rightarrow \tau$ as $t \rightarrow \infty$, we see that, for any $\epsilon>0$, there is $t_{0}>0$ large enough such that $Q\left(t_{0}\right) \in(\tau-\epsilon / 4, \tau+\epsilon / 4)$. Therefore, there exists $n_{0}>0$ such that $Q_{n}\left(t_{0}\right) \in(\tau-\epsilon / 2, \tau+\epsilon / 2)$ for all $n \geq n_{0}$. Passing to a subsequence, we can find $t_{n} \rightarrow \infty$ such that

$$
Q_{n}\left(t_{n}\right) \leq \tau+\epsilon / 2
$$

Moreover, there exists $\left\{y_{n}\right\} \subset \mathbb{R}^{N}$ such that

$$
\int_{B_{t_{0}}\left(y_{n}\right)}\left[\left|\nabla u_{n}\right|^{2}+u_{n}^{2}\right] d x \in(\tau-\epsilon / 2, \tau+\epsilon / 2) .
$$

Let $\psi, \varphi \in C^{1}\left(\mathbb{R}^{N}\right)$ be cut-off functions such that $0 \leq \psi, \varphi \leq 1, \psi \equiv 0$ if $|x| \geq 2$, $\psi \equiv 1$ if $|x| \leq 1, \varphi \equiv 1$ if $|x| \geq 2$, and $\varphi \equiv 0$ if $|x| \leq 1$.

Define $\psi_{n}(\cdot)=\psi\left(\frac{-y_{n}}{R_{1}}\right)$, where $R_{1} \geq t_{0}$ is a number to be determined later, and $\varphi_{n}(\cdot)=\varphi\left(\frac{\cdot y_{n}}{t_{n}}\right)$. Then for $n \geq n_{0}$,

$$
\begin{aligned}
&\left|\int_{\mathbb{R}^{N}}\left[\psi_{n}^{2}\left|\nabla u_{n}\right|^{2}-\left|\nabla\left(\psi_{n} u_{n}\right)\right|^{2}\right] d x\right|=\left|\int_{\mathbb{R}^{N}}\left[\psi_{n}^{2}\left|\nabla u_{n}\right|^{2}-\left|\psi_{n} \nabla u_{n}+\frac{1}{R_{1}} u_{n} \nabla \psi\right|^{2}\right] d x\right| \\
& \leq \frac{C}{R_{1}}, \\
&\left|\int_{\mathbb{R}^{N}}\left[\psi_{n}^{2}\left|\nabla u_{n}\right|^{2}-\nabla u_{n} \nabla\left(\psi_{n}^{2} u_{n}\right)\right] d x\right|=\frac{2}{R_{1}}\left|\int_{\mathbb{R}^{N}} u_{n} \psi_{n} \nabla u_{n} \nabla \psi_{n} d x\right| \leq \frac{C}{R_{1}} .
\end{aligned}
$$


Choosing $R_{1} \geq t_{0}$ large enough such that $C / R_{1}<\epsilon$, we have

$$
\begin{array}{r}
\left|\int_{\mathbb{R}^{N}}\left[\psi_{n}^{2}\left|\nabla u_{n}\right|^{2}-\left|\nabla\left(\psi_{n} u_{n}\right)\right|^{2}\right] d x\right|<\epsilon, \\
\left|\int_{\mathbb{R}^{N}}\left[\psi_{n}^{2}\left|\nabla u_{n}\right|^{2}-\nabla u_{n} \nabla\left(\psi_{n}^{2} u_{n}\right)\right] d x\right|<\epsilon .
\end{array}
$$

By (F1), (F2) and the definition of $\psi$, we get

$$
\begin{aligned}
& \left|\int_{\mathbb{R}^{N}}\left[\psi_{n}^{2} u_{n} f_{\lambda}\left(x, u_{n}\right)-\psi_{n} u_{n} f_{\lambda}\left(x, \psi_{n} u_{n}\right)\right] d x\right| \\
& =\left|\int_{\mathbb{R}^{N}}\left[\psi_{n}^{2} u_{n} f\left(u_{n}\right)-\psi_{n} u_{n} f\left(\psi_{n} u_{n}\right)\right] d x\right| \\
& =\left|\int_{R_{1} \leq\left|x-y_{n}\right| \leq 2 R_{1}}\left[\psi_{n}^{2} u_{n} f\left(u_{n}\right)-\psi_{n} u_{n} f\left(\psi_{n} u_{n}\right)\right] d x\right| \\
& \leq C \int_{R_{1} \leq\left|x-y_{n}\right| \leq 2 R_{1}}\left[\left|\nabla u_{n}\right|^{2}+u_{n}^{2}\right] d x \leq C \epsilon \quad \text { by 6.1] and 6.2. }
\end{aligned}
$$

Similarly, for $n$ large enough, we also have,

$$
\begin{array}{r}
\left|\int_{\mathbb{R}^{N}}\left[\varphi_{n}^{2}\left|\nabla u_{n}\right|^{2}-\left|\nabla\left(\varphi_{n} u_{n}\right)\right|^{2}\right] d x\right|<\mu(\epsilon), \\
\left|\int_{\mathbb{R}^{N}}\left[\varphi_{n}^{2}\left|\nabla u_{n}\right|^{2}-\nabla u_{n} \nabla\left(\varphi_{n}^{2} u_{n}\right)\right] d x\right|<\mu(\epsilon), \\
\left|\int_{\mathbb{R}^{N}}\left[\varphi_{n}^{2} u_{n} f_{\lambda}\left(x, u_{n}\right)-\varphi_{n} u_{n} f_{\lambda}\left(x, \varphi_{n} u_{n}\right)\right] d x\right|<\mu(\epsilon) ;
\end{array}
$$

here and in what follows, we denote by $\mu(\epsilon)$ a quantity which tends to 0 as $\epsilon \rightarrow 0^{+}$.

Let $u_{n}^{1}=\psi_{n} u_{n}, u_{n}^{2}=\varphi_{n} u_{n}$ (note that $u_{n}^{2}$ is not $\left(u_{n}\right)^{2}$ ). Without loss of generality, we assume $t_{n}>R_{1}$. Then by 6.1 and (6.2) we have

$$
\begin{aligned}
\left\|u_{n}-u_{n}^{1}-u_{n}^{2}\right\| & \leq \mu(\epsilon), \\
\left|\int_{\mathbb{R}^{N}}\left(\left|\nabla u_{n}\right|^{2}-\left|\nabla u_{n}^{1}\right|^{2}-\left|\nabla u_{n}^{2}\right|^{2}\right) d x\right| & \leq \mu(\epsilon), \\
\left|\int_{\mathbb{R}^{N}}\left(\left|u_{n}\right|^{2}-\left|u_{n}^{1}\right|^{2}-\left|u_{n}^{2}\right|^{2}\right) d x\right| & \leq \mu(\epsilon) .
\end{aligned}
$$

Noticing that $\left\|u_{n}\right\|^{2} \stackrel{n}{\rightarrow} \ell$, by the definitions of $u_{n}^{1}$ and $u_{n}^{2}$, using 6.2 and 6.9 we see that

$$
\left|\left\|u_{n}^{1}\right\|^{2}-\tau\right| \leq \mu(\epsilon), \quad\left|\left\|u_{n}^{2}\right\|^{2}-(\ell-\tau)\right| \leq \mu(\epsilon) .
$$

From 6.6- 6.8 , we get

$$
\left|\left\langle I^{\prime}\left(u_{n}^{2}\right), u_{n}^{2}\right\rangle-\left\langle I^{\prime}\left(u_{n}\right), \varphi_{n}^{2} u_{n}\right\rangle\right| \leq \mu(\epsilon) .
$$


Since $\left\|I^{\prime}\left(u_{n}\right)\right\|_{H^{-1}\left(\mathbb{R}^{N}\right)} \stackrel{n}{\rightarrow} 0$ and $\left\{u_{n}\right\}$ is bounded in $H^{1}\left(\mathbb{R}^{N}\right)$, we have $\left\langle I^{\prime}\left(u_{n}\right), \varphi_{n}^{2} u_{n}\right\rangle$ $\stackrel{n}{\rightarrow} 0$. Therefore, we get

$$
\int_{\mathbb{R}^{N}}\left[\left|\nabla u_{n}^{2}\right|^{2}+\left|u_{n}^{2}\right|^{2}-f_{\lambda}\left(x, u_{n}^{2}\right) u_{n}^{2}\right] d x=\mu(\epsilon)+o(1) .
$$

Similarly, it follows from $6.3-6.5$ that

$$
\int_{\mathbb{R}^{N}}\left[\left|\nabla u_{n}^{1}\right|^{2}+\left|u_{n}^{1}\right|^{2}-f_{\lambda}\left(x, u_{n}^{1}\right) u_{n}^{1}\right] d x=\mu(\epsilon)+o(1) .
$$

As in the derivation of (6.5), we have

$$
\begin{aligned}
& \left|\int_{\mathbb{R}^{N}}\left[\psi_{n} F_{\lambda}\left(x, u_{n}\right)-F_{\lambda}\left(x, u_{n}^{1}\right)\right] d x\right| \leq \mu(\epsilon), \\
& \left|\int_{\mathbb{R}^{N}}\left[\varphi_{n} F_{\lambda}\left(x, u_{n}\right)-F_{\lambda}\left(x, u_{n}^{2}\right)\right] d x\right| \leq \mu(\epsilon) .
\end{aligned}
$$

Therefore,

$$
\left|\int_{\mathbb{R}^{N}}\left[F_{\lambda}\left(x, u_{n}\right)-F_{\lambda}\left(x, u_{n}^{1}\right)-F_{\lambda}\left(x, u_{n}^{2}\right)\right] d x\right| \leq \mu(\epsilon) .
$$

It follows from 6.10, 6.11 and 6.15) that

$$
\left|I\left(u_{n}\right)-I\left(u_{n}^{1}\right)-I\left(u_{n}^{2}\right)\right| \leq \mu(\epsilon) .
$$

Thus,

$$
I\left(u_{n}\right) \geq I\left(u_{n}^{1}\right)+I\left(u_{n}^{2}\right)-\mu(\epsilon) .
$$

Since $\operatorname{dist}\left(\operatorname{supp}\left\{u_{n}^{1}\right\}, \operatorname{supp}\left\{u_{n}^{2}\right\}\right) \stackrel{n}{\rightarrow} \infty$, without loss of generality we may assume that $\operatorname{dist}\left(\operatorname{supp}\left\{u_{n}^{2}\right\},\{0\}\right) \stackrel{n}{\rightarrow} \infty$. Hence for any given $R>0$ and $n$ large enough we have $\operatorname{supp}\left\{u_{n}^{2}\right\} \cap B_{R}(0)=\emptyset$. Therefore, from $g(x) \rightarrow 1$ as $|x| \rightarrow \infty$, we deduce that

$$
\begin{aligned}
\left|I\left(u_{n}^{2}\right)-I^{\infty}\left(u_{n}^{2}\right)\right| & =\left|\int_{\mathbb{R}^{N}}\left[f_{\lambda}\left(x, u_{n}^{2}\right) u_{n}^{2}-\bar{f}_{\lambda}\left(u_{n}^{2}\right) u_{n}^{2}\right] d x\right| \\
& =\left.\lambda\left|\int_{\mathbb{R}^{N}}(g(x)-1)\right| u_{n}^{2}\right|^{2} d x \mid \stackrel{n}{\rightarrow} 0 .
\end{aligned}
$$

By 6.16 and 6.17),

$$
I\left(u_{n}\right) \geq I\left(u_{n}^{1}\right)+I^{\infty}\left(u_{n}^{2}\right)-\mu(\epsilon)+o(1) .
$$

Let $v_{n}(x)=u_{n}^{2}(\sigma x)$, so that

$$
\left\langle I^{\infty \prime}\left(v_{n}\right), v_{n}\right\rangle=\sigma^{-n}\left(\sigma^{2}-1\right) \int_{\mathbb{R}^{N}}\left|\nabla u_{n}^{2}\right|^{2} d x+\sigma^{-n}+\epsilon_{n},
$$


where $\epsilon_{n}=\int_{\mathbb{R}^{N}}\left[\left|\nabla u_{n}^{2}\right|^{2}+\left|u_{n}^{2}\right|^{2}-\bar{f}_{\lambda}\left(u_{n}^{2}\right) u_{n}^{2}\right] d x$. Using 6.13) and 6.17, we have

$$
\epsilon_{n}=\mu(\epsilon)+o(1)
$$

We claim that there is a sequence $\left\{\sigma_{n}\right\}$ with $\sigma_{n} \stackrel{n}{\rightarrow} 1$ such that

$$
\left(\sigma_{n}^{2}-1\right) \int_{\mathbb{R}^{N}}\left|\nabla u_{n}^{2}\right|^{2} d x+\epsilon_{n}=0 .
$$

To prove this, we need only show that there is a constant $A>0$ such that

$$
\int_{\mathbb{R}^{N}}\left|\nabla u_{n}^{2}\right|^{2} d x \geq A>0 \quad \text { for } n \text { large. }
$$

By contradiction, we suppose that 6.21 is false. Then there exists a subsequence of $\left\{u_{n}^{2}\right\}$, still denoted by $\left\{u_{n}^{2}\right\}$, such that

$$
\lim _{n \rightarrow \infty} \int_{\mathbb{R}^{N}}\left|\nabla u_{n}^{2}\right|^{2} d x=0 .
$$

From 6.13), (F1) and (F2), we have

$$
\begin{aligned}
\left\|u_{n}^{2}\right\|^{2} & =-\lambda \int_{\mathbb{R}^{N}} g(x)\left|u_{n}^{2}\right|^{2} d x+\int_{\mathbb{R}^{N}} f\left(u_{n}^{2}\right) u_{n}^{2} d x+\mu(\epsilon)+o(1) \\
& \leq \frac{1}{2} \int_{\mathbb{R}^{N}}\left|u_{n}^{2}\right|^{2} d x+C\left(\int_{\mathbb{R}^{N}}\left|\nabla u_{n}^{2}\right|^{2} d x\right)^{N /(N-2)}+\mu(\epsilon)+o(1) .
\end{aligned}
$$

Then 6.22 and 6.23 imply that $\left\|u_{n}^{2}\right\|^{2} \leq \mu(\epsilon)+o(1)$, which contradicts the second inequality of (6.12).

Therefore, we can find $\sigma_{n}=1+\mu(\epsilon)+o(1)$ such that $v_{n} \in \Lambda$ (see 4.18) and

$$
I^{\infty}\left(v_{n}\right)=I^{\infty}\left(u_{n}^{2}\right)+\mu(\epsilon)+o(1) .
$$

This implies that

$$
I^{\infty}\left(u_{n}^{2}\right)=I^{\infty}\left(v_{n}\right)-\mu(\epsilon)+o(1) \geq J^{\infty}-\mu(\epsilon)+o(1) .
$$

By (F1) and (F3),

$$
\begin{aligned}
I\left(u_{n}^{1}\right) & =\frac{1}{2} \int_{\mathbb{R}^{N}}\left[\left|\nabla u_{n}^{1}\right|^{2}+(1+\lambda g(x))\left|u_{n}^{1}\right|^{2}\right] d x-\int_{\mathbb{R}^{N}} F\left(u_{n}^{1}\right) d x \\
& \geq \frac{1}{2} \int_{\mathbb{R}^{N}}\left[\left|\nabla u_{n}^{1}\right|^{2}+\left|u_{n}^{1}\right|^{2}-f_{\lambda}\left(x, u_{n}^{1}\right) u_{n}^{1}\right] d x \\
& =\mu(\epsilon)+o(1) \quad \text { by } 6.14 .
\end{aligned}
$$

Then from 6.18, 6.24 and 6.25, we get

$$
I\left(u_{n}\right) \geq J^{\infty}-\mu(\epsilon)+o(1) .
$$


This implies $\lim _{n} I\left(u_{n}\right)=c \geq J^{\infty}$, which contradicts $c<J^{\infty}$. So, Case (B) cannot occur.

Finally, we know that only compactness can occur, that is, $\tau=\ell$. So, by the second inequality of 6.12 there exist $\left\{y_{n}\right\} \subset \mathbb{R}^{N}$ and a subsequence of $\left\{u_{n}\right\}$ (still denoted by $\left.\left\{u_{n}\right\}\right)$ such that for any $\epsilon>0$, there is $R=R(\epsilon)>0$ such that

$$
\int_{\left|x-y_{n}\right| \geq R}\left[\left|\nabla u_{n}\right|^{2}+u_{n}^{2}\right] d x<\epsilon .
$$

If $\left\{y_{n}\right\}$ is bounded, then there exists $\tilde{R}>0$ such that

$$
\int_{|x| \geq \tilde{R}}\left[\left|\nabla u_{n}\right|^{2}+u_{n}^{2}\right] d x \leq \int_{\left|x-y_{n}\right| \geq R}\left[\left|\nabla u_{n}\right|^{2}+u_{n}^{2}\right] d x<\epsilon,
$$

and 4.15$)$ is proved.

If $\left|y_{n}\right| \stackrel{n}{\rightarrow} \infty$, noticing that $g(x) \rightarrow 1$ as $|x| \rightarrow \infty$, it follows from 6.26 that

$$
\begin{aligned}
I\left(u_{n}\right) & =I^{\infty}\left(u_{n}\right)-\lambda \int_{\mathbb{R}^{N}}(1-g(x))\left|u_{n}\right|^{2} d x \\
& =I^{\infty}\left(u_{n}\right)-\lambda\left(\int_{\left|x-y_{n}\right| \geq R}+\int_{\left|x-y_{n}\right| \leq R}\right)(1-g(x))\left|u_{n}\right|^{2} d x \\
& \geq I^{\infty}\left(u_{n}\right)-C \epsilon+o(1) .
\end{aligned}
$$

Similar to the proof of 6.24, we can find $\sigma_{n}$ with $\sigma_{n}=1+\mu(\epsilon)+o(1)$ and $v_{n}(x)=$ $u_{n}\left(\sigma_{n} x\right) \in \Lambda$ such that

$$
I^{\infty}\left(u_{n}\right)=I^{\infty}\left(v_{n}\right)-\mu(\epsilon)+o(1) \geq J^{\infty}-\mu(\epsilon)+o(1) .
$$

Using 6.27) and 6.28), we have

$$
I\left(u_{n}\right) \geq J^{\infty}-\mu(\epsilon)+o(1) .
$$

This implies $c \geq J^{\infty}$, a contradiction again.

Acknowledgments. The authors express their sincere thanks to Professor C. A. Stuart for his helpful comments. Thanks also to the referee for some valuable suggestions. This work was supported by NSFC grants No.10571174 and 10631030, and CAS grant KJCX3-SYW-S03.

\section{References}

[1] Bartsch, T., Pankov, A., Wang, Z. Q.: Nonlinear Schrödinger equations with steep potential well. Comm. Contemp. Math. 3, 549-569 (2001) Zbl 1076.35037| MR 1869104

[2] Bartsch, T., Wang, Z. Q.: Existence and multiplicity results for some superlinear elliptic problems on $\mathbb{R}^{N}$. Comm. Partial Differential Equations 20, 1725-1741 (1995) Zbl 0837.35043 MR 1349229

[3] Bartsch, T., Wang, Z. Q.: Multiple positive solutions for a nonlinear Schrödinger equation. Z. Angew. Math. Phys. 51, 366-384 (2000) Zbl 0972.35145 MR 1762697 
[4] Berestycki, H., Lions, P.-L.: Nonlinear scalar field equations, I. Existence of a ground state. Arch. Ration. Mech. Anal. 82, 313-346 (1983) Zbl 0533.35029 MR 0695535

[5] Byeon, J., Wang, Z. Q.: Spherical semiclassical states of a critical frequency for Schrödinger equations with decaying potentials. J. Eur. Math. Soc. 8, 217-228 (2006) Zbl pre05053360 MR 2239273

[6] Chen, Z. H., Shen, Y. T., Yao, Y. X.: Some existence results of solutions for $p$-Laplacian. Acta Math. Sci. Ser. B 23, 487-496 (2003) Zbl 1119.35320 MR 2032552

[7] Clapp, M., Ding, Y. H.: Minimal nodal solutions of a Schrödinger equation with critical nonlinearity and symmetric potential. Differential Integral Equations 16, 981-992 (2003) Zbl pre02004935 MR 1989597

[8] Costa, D., Tehrani, H.: On a class of asymptotically linear elliptic problems in $\mathbb{R}^{N}$. J. Differential Equations 173, 470-494 (2001) Zbl 1098.35526 MR 1834123

[9] Del Pino, M., Felmer, P.: Local mountain passes for semilinear elliptic problems in unbounded domains. Calc. Var. Partial Differential Equations 4, 121-137 (1996) Zbl 0844.35032 MR 1379196

[10] Ding, Y. H., Tanaka, K.: Multiplicity of positive solutions of a nonlinear Schrödinger equation. Manuscripta Math. 112, 109-135 (2003) Zbl 1038.35114 MR 2005933

[11] Ekeland, I.: Convexity Methods in Hamiltonian Mechanics. Springer (1990) Zbl 0707.70003 MR 1051888

[12] de Figueiredo, D. G., Ding, Y. H.: Solutions of a nonlinear Schrödinger equation. Discrete Contin. Dynam. Systems 8, 563-584 (2002) Zbl 1004.35107 MR 1897867

[13] van Heerden, F. A.: Multiple solutions for a Schrödinger type equation with an asymptotically linear term. Nonlinear Anal. 55, 739-758 (2003) Zbl pre02005480 MR 2060527

[14] van Heerden, F. A., Wang, Z. Q.: Schrödinger type equations with asymptotically linear nonlinearities. Differential Integral Equations 16, 257-280 (2003) Zbl 1030.35067 MR 1947953

[15] Jeanjean, L., On the existence of bounded Palais-Smale sequences and applications to a Landesman-Lazer type problem set on $\mathbf{R}^{N}$. Proc. Roy. Soc. Edinburgh Sect. A 129, 787 809 (1999) Zbl 0935.35044 MR 1718530

[16] Jeanjean, L., Tanaka, K.: A positive solution for an asymptotically linear elliptic problem on $\mathbf{R}^{N}$ autonomous at infinity. ESAIM Control Optim. Calc. Var. 7, 597-614 (2002) Zbl pre01966590 MR 1925042

[17] Jeanjean, L., Tanaka, K.: Singularly perturbed elliptic problems with superlinear or asymptotically linear nonlinearities. Calc. Var. Partial Differential Equations 21, 287-318 (2004) Zbl 1060.35012 MR 2094325

[18] Li, G. B., Zhou, H. S.: The existence of a positive solution to asymptotically linear scalar field equations. Proc. Roy. Soc. Edinburgh Sect. A 130, 81-105 (2000) Zbl 0942.35075 MR 1742582

[19] Li, Y. Q., Wang, Z. Q., Zeng, J.: Ground states of nonlinear Schrödinger equations with potentials. Ann. Inst. H. Poincaré Anal. Non Linéaire 23, 829-837 (2006) Zbl 1111.35079 MR 2271695

[20] Lions, P.-L.: The concentration-compactness principle in the calculus of variations. The locally compact case. Parts 1 and 2. Ann. Inst. H. Poincaré Anal. Non Linéaire 1, 109-145 and 223-283 (1984) Zbl 0541.49009 (part 1), Zbl 0704.49004(part 2) MR 0778970 (part 1), MR 0778974 (part 2)

[21] Liu, C. Y., Wang, Z. P., Zhou, H. S.: Asymptotically linear Schrödinger equation with potential vanishing at infinity. J. Differential Equations 245, 201-222 (2008) doi:10.1016/j.jde.2008.01.006 
[22] Liu, Z. L., van Heerden, F. A., Wang, Z. Q.: Nodal type bounded states of Schrödinger equations via invariant set and minimax methods. J. Differential Equations 214, 358-390 (2005) Zbl pre02189408 MR 2145254

[23] Liu, Z. L., Su, J. B., Weth, T.: Compactness results for Schrödinger equations with asymptotically linear terms. J. Differential Equations 231, 501-512 (2006) Zbl pre05115328 MR 2287894

[24] Liu, Z. L., Wang, Z. Q.: Existence of a positive solution of an elliptic equation on $\mathbb{R}^{N}$. Proc. Roy. Soc. Edinburgh Sect. A 134, 191-200 (2004) Zbl 1067.35029 MR 2039911

[25] Rabinowitz, P. H.: On a class of nonlinear Schrödinger equations. Z. Angew. Math. Phys. 43, 270-290 (1992) Zbl 0763.35087 MR 1162728

[26] Schechter, M.: A variation of the mountain pass lemma and applications. J. London Math. Soc. 44, 491-502 (1991) Zbl 0756.35032 MR 1149010

[27] Strauss, W. A.: Existence of solitary waves in higher dimensions. Comm. Math. Phys. 55, 149-162 (1977) Zbl 0356.35028 MR 0454365

[28] Stuart, C. A.: A note on Maxwell equation. Preprint

[29] Stuart, C. A., Zhou, H. S.: A variational problem related to self-trapping of an electromagnetic field. Math. Methods Appl. Sci. 19, 1397-1407 (1996) Zbl 0862.35123 MR 1414401

[30] Stuart, C. A., Zhou, H. S.: Applying the mountain pass theorem to an asymptotically linear elliptic equation on $\mathbb{R}^{N}$. Comm. Partial Differential Equations 24, 1731-1758 (1999) Zbl 0935.35043 MR 1708107

[31] Stuart, C. A., Zhou, H. S.: Global branch of solutions for nonlinear Schrödinger equations with deepening potential well. Proc. London Math. Soc. 92, 655-681 (2006) Zbl pre05025086 MR 2223540

[32] Stuart, C. A., Zhou, H. S.: Positive eigenfunctions of a Schrödinger operator. J. London Math. Soc. 72, 429-441 (2005) Zbl 1095.35020 MR 2156662

[33] Tehrani, H.: Existence results for an indefinite unbounded perturbation of a resonant Schrödinger equation. J. Differential Equations 236, 1-28 (2007) Zbl 1122.35139 MR 2319918

[34] Zhou, H. S., Zhu, H. B.: Asymptotically linear elliptic problem on $\mathbb{R}^{N}$. Quart. J. Math. 59, 523-541 (2008) doi:10.1093/qmath/ham047

[35] Zhu, X. P., Cao, D. M.: The concentration-compactness principle in nonlinear elliptic equations. Acta Math. Sci. 9, 307-328 (1989) Zbl 0702.35095 |MR 1043058 